\title{
14. RADIOLARIANS FROM THE NORTHWEST PACIFIC, DEEP SEA DRILLING PROJECT LEG 86 ${ }^{1}$
}

\author{
Joseph J. Morley, Lamont-Doherty Geological Observatory of Columbia University²
}

\begin{abstract}
Radiolarians occur in sediments from all northwest Pacific sites drilled on DSDP Leg 86. The oldest radiolarianbearing sediment was recovered at Site 581 , where middle Miocene radiolarians were found. Site 578 recovered late Miocene through Pleistocene radiolarians, while an early Pliocene through Pleistocene radiolarian assemblage was present in sediments at Sites 577 and 579. Site 580 contained radiolarians ranging in age from late Pliocene to Pleistocene. Only Pleistocene radiolarians were preserved in sediments from Site 576.

The onset of Miocene biosiliceous sedimentation in the northwest Pacific is not synchronous, but is dependent upon the time when each specific site exited from the low-productivity central gyre into what appears to be a preexisting, high-productivity gyre-margin region. During this Neogene and Quaternary period of biosiliceous sedimentation in the northwest Pacific, radiolarian productivity increased throughout the early and middle late Pliocene, reaching a maximum between 2.3 and 2.4 m.y. ago. Early Pleistocene productivity levels are lower than late Pliocene values, with indications of minor pulses in the early, middle, and latest Pleistocene.
\end{abstract}

\section{INTRODUCTION}

Deep Sea Drilling Project (DSDP) Leg 86 drilled 11 holes at six sites in the northwest Pacific (Table 1, Fig. 1). The six sites were positioned so that the radiolarians preserved in the sediments from these sites reflected Miocene through Pleistocene conditions in the northwest Pacific along a transect across the Subarctic Convergence, with its end members centered in the southern subarctic gyre and the central subtropical gyre.

Although investigators (Kling, 1971; Ling, 1973; Foreman, 1973a; Foreman, 1975; Ling, 1980; Sakai, 1980; Reynolds, 1980; Schaaf, 1981) have reported on radiolarians in sediments from earlier northwest Pacific DSDP Legs $(6,19,20,32,55,56,57$, and 62$)$, the sites drilled during Leg 86 represent the first time that sediments from this region have been recovered using the hydraulic piston corer. This technique was utilized at all sites except Site 581, where sediments were recovered using the rotary drill. The relatively undisturbed sediment sequences from these various sites provide a rigorous test of the siliceous faunal biostratigraphv for the North Pacific first proposed by Hays (1970) and then modified by Foreman (1975).

Radiolarians occur at all sites drilled on Leg 86. At Site 581, radiolarians range in age from middle Miocene to Pleistocene. Site 578 contains radiolarians characteristic of the late Miocene through Pleistocene. Radiolarians of early Pliocene to Pleistocene age are present at Sites 577 and 579. A late Pliocene to Pleistocene radiolarian assemblage is found in sediments at Site 580 . Radiolarians at Site 576 are limited to the Pleistocene.

\footnotetext{
${ }^{1}$ Heath, G. R., Burckle, L. H., et al., Init. Repts. DSDP, 86: Washington (U.S. Govt. Printing Office)

2 Address: Lamont-Doherty Geological Observatory of Columbia University, Palisades,
} NY 10964.
Table 1. Coordinates and water depths of DSDP Leg 86 sites.

\begin{tabular}{lccc}
\hline Hole & Latitude & Longitude & $\begin{array}{c}\text { Water } \\
\text { depth (m) }\end{array}$ \\
\hline 576 & $32^{\circ} 21.36^{\prime} \mathrm{N}$ & $164^{\circ} 16.54^{\prime} \mathrm{E}$ & 6217 \\
$576 \mathrm{~A}$ & $32^{\circ} 21.38^{\prime} \mathrm{N}$ & $164^{\circ} 16.52^{\prime} \mathrm{E}$ & 6217 \\
$576 \mathrm{~B}$ & $32^{\circ} 21.37^{\prime} \mathrm{N}$ & $164^{\circ} 16.52^{\prime} \mathrm{E}$ & 6217 \\
577 & $32^{\circ} 26.51^{\prime} \mathrm{N}$ & $157^{\circ} 43.40^{\prime} \mathrm{E}$ & 2675 \\
$577 \mathrm{~A}$ & $32^{\circ} 26.53^{\prime} \mathrm{N}$ & $157^{\circ} 43.39^{\prime} \mathrm{E}$ & 2675 \\
$577 \mathrm{~B}$ & $32^{\circ} 26.48^{\prime} \mathrm{N}$ & $157^{\circ} 43.39^{\prime} \mathrm{E}$ & 2675 \\
578 & $33^{\circ} 55.56^{\prime} \mathrm{N}$ & $151^{\circ} 37.74^{\prime} \mathrm{E}$ & 6010 \\
579 & $38^{\circ} 37.68^{\prime} \mathrm{N}$ & $153^{\circ} 50.17^{\prime} \mathrm{E}$ & 5737 \\
$579 \mathrm{~A}$ & $38^{\circ} 37.61^{\prime} \mathrm{N}$ & $153^{\circ} 50.28^{\prime} \mathrm{E}$ & 5737 \\
580 & $41^{\circ} 37.47^{\prime} \mathrm{N}$ & $153^{\circ} 58.58^{\prime} \mathrm{E}$ & 5375 \\
581 & $43^{\circ} 55.62^{\prime} \mathrm{N}$ & $159^{\circ} 47.76^{\prime} \mathrm{E}$ & 5476 \\
\hline
\end{tabular}

\section{PROCEDURES AND ANALYSES}

Up to three samples were taken per core for biostratigraphic analysis. After disaggregation, the sample was sieved at $63 \mu \mathrm{m}$. The coarse fraction from sediments at Sites 576 and 577 was strewn on slides. The greater than $63 \mu \mathrm{m}$ fraction from Sites $578,579,580$, and 581 was randomly settled onto slides following the technique described by Moore (1973).

Radiolarian preservation is indicated for samples taken at Sites 577 through 581 (Tables 2-6) with abbreviations defined as follows: G (good), signifying that more than half of the specimens are unbroken with minimal dissolution; $\mathbf{M}$ (moderate), denoting breakage in more than half the specimens and/or some degree of dissolution; and P (poor), meaning that most specimens were fragmented and/or exhibited a high degree of dissolution. Because samples from Sites 578 through 581 were dried and weighed prior to disaggregation, and slides prepared using the random settling technique, it is possible to calculate the relative abundance of radiolarians per gram of sediment. The abundance values in Tables 3 through 6 are rounded to the nearest thousand radiolarians per gram.

The relative abundance of individual species given in Tables 2 through 6 is based on examination of a minimum of 500 radiolarians per sample with abbreviations defined as follows: $r$, very rare $(0.01-$ $0.10 \%) ; \mathrm{R}$, rare $(0.11-1.0 \%)$; F, few $(1.1-10.0 \%)$; C, common (10.1$30.0 \%) ;+$, one or two specimens present; - , specimens sought but not found. 


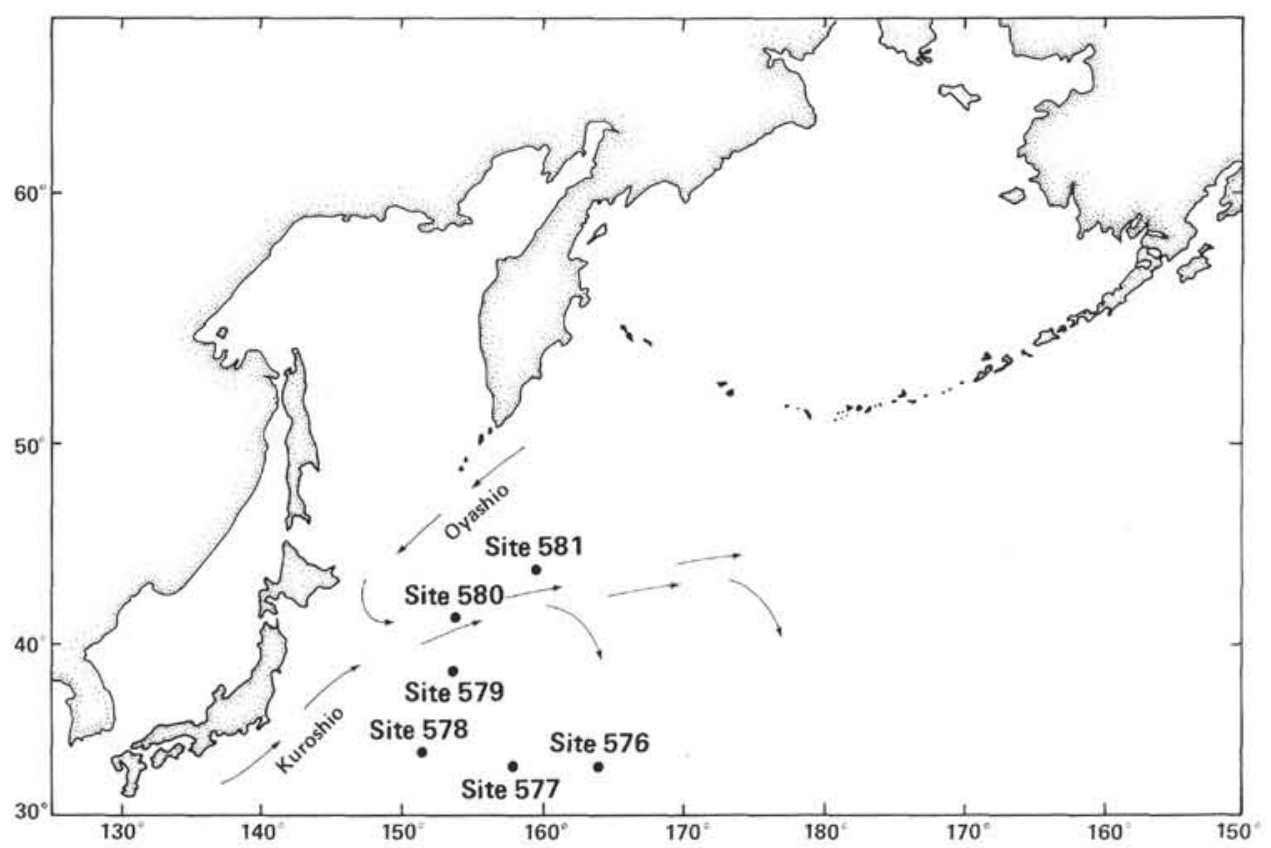

Figure 1. Location of DSDP Leg 86 sites.

\section{BIOSTRATIGRAPHIC FRAMEWORK}

The radiolarian zonation proposed by Foreman (1975) was applied to the radiolarian assemblage in the Pliocene through Pleistocene sediment sequence recovered during Leg 86. For the middle to late Miocene radiolarian sequences, the zonation defined by Riedel and Sanfilippo (1978) was appropriate for the radiolarian fauna present. The sites drilled on this leg spanned a wide geographic area of the northwest Pacific, sampling a subtropical to subarctic fauna. Even so, this stratigraphic zonation, with its various datum levels, was easily applied to the sediment sequence from each hole, providing a uniform biostratigraphic framework. The definitions of these zones are as follows:

Botryostrobus aquilonaris Zone, Hays, 1970

The base is defined by the presence of Botryostrobus aquilonaris (=Eucyrtidium tumidulum of Hays, $1970 ;=$ Artostrobium miralestense of Kling, 1973; = Artostrobium tumidulum of Foreman, 1975 ) and the absence of Stylatractus universus (= Axoprunum angelium of Foreman, 1975). The age of the base of this zone is $0.425 \mathrm{~m} . \mathrm{y}$. based on the estimated age of the upper limit of Stylatractus universus (Hays and Shackleton, 1976; Morley and Shackleton, 1978).

Stylatractus universus Zone, Hays, 1970

The base of this zone is defined by the extinction of Eucyrtidium matuyamai and the top by the upper limit of Stylatractus universus. The age range of this zone is from 0.97 to $0.425 \mathrm{~m} . \mathrm{y}$.

Eucyrtidium matuyamai Zone, Hays, 1970, emend. Foreman, 1973a This zone is defined by the range of Eucyrtidium matuyamai and spans the period from 1.88 to 0.97 m.y.

Lamprocyrtis heteroporos Zone, Hays, 1970, emend. Foreman, 1975 The base of this zone is marked by the extinction of Stichocorys peregrina, and the top is defined by the earliest evolutionary appearance of Eucyrtidium matuyamai. The zone ranges in age from 2.85 to $1.88 \mathrm{~m} . \mathrm{y}$.

Sphaeropyle langii Zone, Foreman, 1975

The base is defined as the first morphological appearance of Sphaeropyle langii, and the top is marked by the upper limit of Stichocorys peregrina. The age range of this zone is from 4.40 to $2.85 \mathrm{~m}$.y.
Stichocorys peregrina Zone, Riedel and Sanfilippo, 1970, emend. Foreman, 1975

The base is defined by the first evolutionary appearance of Stichocorys peregrina and the top by the earliest morphological appearance of Sphaeropyle langii. This zone spans the time interval from 6.20 to 4.40 m.y.

Didymocyrtis penultima Zone, Riedel and Sanfilippo, 1970

The base is defined by the first evolutionary appearance of Didymocyrtis penultima ( = Ommatartus penultimus of Riedel and Sanfilippo, 1970) and the top by the first evolutionary appearance of Stichocorys peregrina. The age range of this zone is from 7.90 to 6.20 m.y.

Didymocyrtis antepenultima Zone, Riedel and Sanfilippo, 1970, emend. Riedel and Sanfilippo, 1978

The base of this zone is defined by the earliest evolutionary appearance of Didymocyrtis antepenultima (=Ommatartus antepenultimus of Riedel and Sanfilippo, 1970), and the top is marked by the first evolutionary appearance of Didymocyrtis penultima. This zone spans the time interval from 10.80 to 7.90 m.y.

Diartus petterssoni Zone, Riedel and Sanfilippo, 1970, emend. Riedel and Sanfilippo, 1978

The base of this zone is marked by the earliest morphologic appearance of Diartus petterssoni ( = Cannartus petterssoni of Riedel and Sanfilippo, 1970). The top of this zone is defined by the first evolutionary appearance of Didymocyrtis antepenultima. The age range of this zone is from 12.50 to 10.80 m.y.

Dorcadospyris alata Zone, Riedel and Sanfilippo, 1970, emend. Riedel and Sanfilippo, 1971

The base of this zone is marked by the first evolutionary appearance of Dorcadospyris alata, and the top is delineated by the earliest morphologic appearance of Diartus petterssoni. This zone spans the time interval from 15.25 to 12.50 m.y.

\section{RADIOLARIAN SITE SUMMARIES}

\section{Site 576}

At Site 576, located between the Shatsky Rise and Emperor Seamounts, sediments from three holes (576, 576A, 576B) were recovered using the hydraulic piston corer. Moderately well-preserved late Pleistocene radiolarians are present in the upper few meters of the pelagic 
clay sequence. The topmost sediments in each of the three holes contain a fauna characteristic of the Botryostrobus aquilonaris Zone, with fauna in the underlying sediments in Core 1 belonging to the Stylatractus universus Zone. Although radiolarians are present in Core 2 from all three holes, the low abundance and poor preservation prevented precise age determination. Radiolarians were absent from sediments in all three holes below Core 2 except for Hole 576 which contained traces of a Pleistocene(?) fauna in Core 3 (Sample 576-3,CC) and an Upper Cretaceous(?) fauna in a thin calcareous sequence in Core 8 (Sample 576-8,CC).

\section{Site 577}

The three holes $(577,577 \mathrm{~A}, 577 \mathrm{~B})$ drilled at Site 577 sampled a calcareous sequence on the western flank of the Shatsky Rise. Radiolarians are present in only the first six cores from Holes 577 (Table 2) and 577A. The well-preserved fauna in the uppermost sediments from Core 1 is representative of the late Pleistocene Botryostrobus aquilonaris Zone. The remainder of Core 1 contains a fauna characteristic of the Stylatractus universus Zone. Core 2 through the middle of Core 3 contains good to poorly preserved early Pleistocene radiolarians of the Eucyrtidium matuyamai Zone. Some difficulty was encountered in locating the base of this zone in Holes 577 and $577 \mathrm{~A}$ because of the very low abundance of $E$. $m a$ tuyamai in Core 3 . Radiolarians are moderately to poorly preserved in Cores 4 through 6 . Late Pliocene radiolarians of the Lamprocyrtis heteroporos Zone are present in sediments from the lower portion of Core 3 through the upper half of Core 4. The remainder of Core 4 through Core 5 contains middle Pliocene radiolarians belonging to the Sphaeropyle langii Zone, with the early Pliocene fauna in Core 6 characteristic of the Stichocorys peregrina Zone.

\section{Site 578}

Hole 578, located west of the Shatsky Rise, sampled a thick pelagic clay sequence overlying Cretaceous chert and chalk. The gray to brown clays in the upper $120 \mathrm{~m}$ of sediment (Cores 1-14) contain radiolarians (Table 3). Radiolarians are not present in the dark brown clays and chert in Cores 15 through 20.

Preservation of the Pleistocene radiolarians in the uppermost sediments from the first eight cores varies from good to poor. Fauna characteristic of the Botryostrobus aquilonaris Zone is present in sediments from the tops of Cores 1 through 3, with the latest occurrence of Stylatractus universus in Sample 578-3-2, 50-51 cm, marking the bottom of this zone (level of datum based on more extensive sampling than shown in Table 3). The remainder of Core 3 and the uppermost sediments in Cores 4 and 5 contain radiolarians representative of the $S$. universus Zone. The presence of Eucyrtidium matuyamai in sediments at the tops of Cores 6, 7, and 8 indicates that the fauna in these sediments is early Pleistocene in age, belonging to the E. matuyamai Zone.

The fauna in the remainder of Core 8 is characteristic of the late Pliocene Lamprocyrtis heteroporos Zone. The top of the Sphaeropyle langii Zone is located between Samples 578-8,CC and 578-10-1, 10-11 cm, as marked by the latest occurrence of Stichocorys peregrina. The boundary between early and late Pliocene, defined by the upper limit of $S$. delmontensis, occurs within Core 10 , with the remainder of Core 11 and the top of Core 12 containing fauna indicative of the middle to lower Sphaeropyle langii Zone. Although not delineated by any siliceous faunal marker, the magnetostratigraphy shows that the Miocene/Pliocene boundary occurs near the base of Core 12 (see Site 578 chapter, this volume). Late Miocene radiolarians representative of the Stichocorys peregrina Zone are present in sediments from Core 13 and the top of Core 14.

\section{Site 579}

Located at the southern limit of the transition zone between subtropical and subarctic gyres, Site 579 contains radiolarians throughout its $150 \mathrm{~m}$ of siliceous clays and oozes (Table 4). Radiolarians are well to moderately preserved in late Pliocene through Pleistocene sediments and moderately to poorly preserved in early Pliocene sediments.

The fauna in Hole 579 and in the uppermost sediment from Core 1 in Hole 579A is indicative of the Botryostrobus aquilonaris Zone. The top of the Stylatractus universus Zone, as marked by the upper limit of $S$. universus, occurs between Samples 579A-1-3, 145-147 $\mathrm{cm}$ and 579A-1-4, 5-7 cm (based on more extensive sampling than shown in Table 4). Radiolarians present in sediments from Core 2 from Hole 579A are also representative of this zone. The Eucyrtidium matuyamai Zone extends from the lower portion of Core 3 through at least the bottom of Core 5 based on the occurrence of $E$. $m a$ tuyamai throughout this sediment sequence. Sediments in Cores 6 through 8 from Hole 579A contain a radiolarian fauna characteristic of the Lamprocyrtis heteroporos Zone, with the bottom of this zone marked by the latest occurrence of Stichocorys peregrina located between Samples 579A-8,CC $(25-27 \mathrm{~cm})$ and 579A-9,CC $(5-7 \mathrm{~cm})$. The upper limit of $S$. delmontensis occurs between Samples 579A-10,CC (15-17 cm) and 579A-11,CC $(5-7 \mathrm{~cm})$, indicating that the boundary between early and late Pliocene is located within Core 11. Radiolarians in sediments in Cores 9 through 12 from Hole 579A are representative of the Sphaeropyle langii Zone, while Cores 13 through 15 from Hole 579A contain an early Pliocene radiolarian fauna belonging to the Stichocorys peregrina Zone.

\section{Site $\mathbf{5 8 0}$}

At Site 580, located near the present-day subarctic front, sediments were recovered to a sub-bottom depth of $155 \mathrm{~m}$. The radiolarians that are present throughout the siliceous clay to clayey diatom ooze sediment sequence are well to moderately preserved (Table 5).

The first three cores contain a late Pleistocene radiolarian fauna representative of the Botryostrobus aquilonaris Zone. Examination of closely spaced samples from Cores 4 through 7 (not all shown in Table 5) shows that 
Table 2. Abundances of some radiolarians from Hole 577.

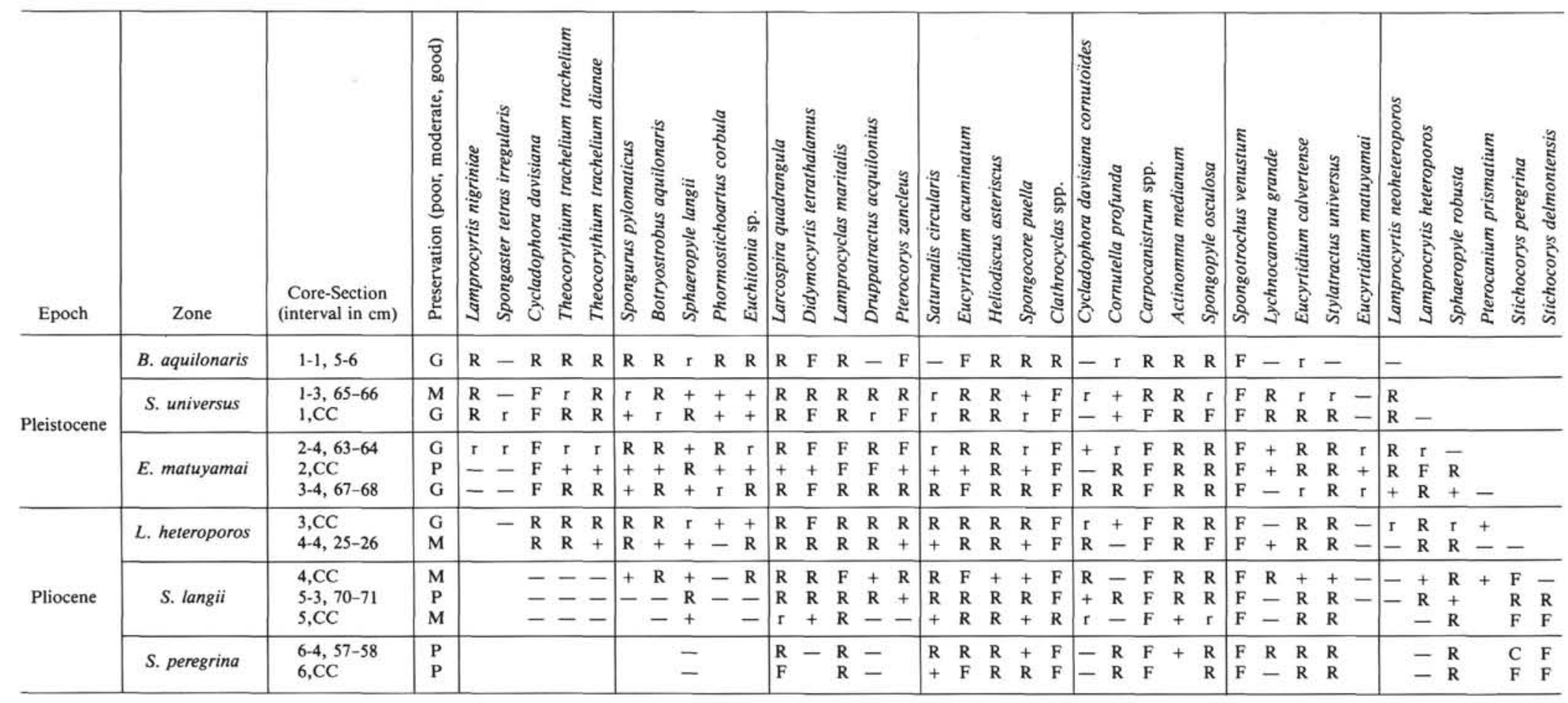

Note: Cores 7-13 are barren of radiolaria. See Procedures and Analyses for explanation of entries. 
Table 3. Abundances of some radiolarians from Hole 578.

\begin{tabular}{|c|c|c|c|c|c|c|c|c|c|c|c|c|c|c|c|c|c|c|c|c|c|c|c|c|c|c|c|c|c|c|c|}
\hline Epoch & Zone & $\begin{array}{c}\text { Core-Section } \\
\text { (interval in cm) }\end{array}$ & $\begin{array}{c}\text { Radiolarian } \\
\text { abundance } \\
\text { (thousands } \\
\text { per gram } \\
\text { of sediment) }\end{array}$ & 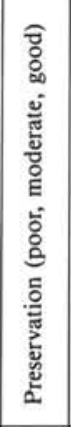 & 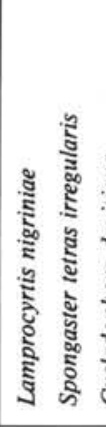 & 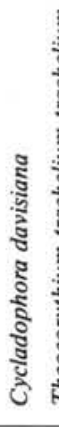 & 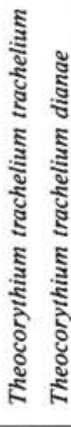 & 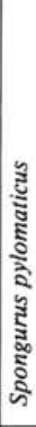 & 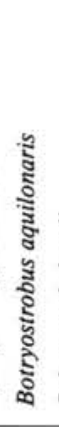 & 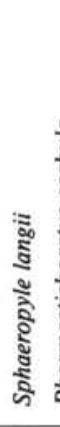 & 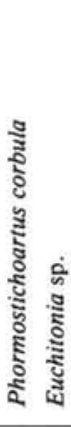 & 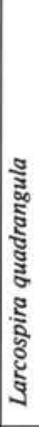 & 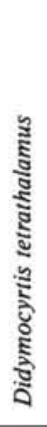 & 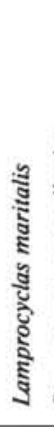 & 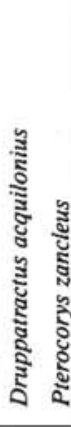 & & 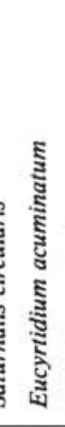 & 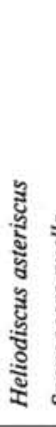 & 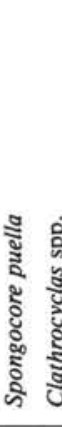 & है & 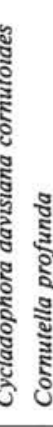 & 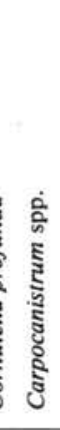 & 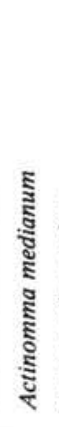 & 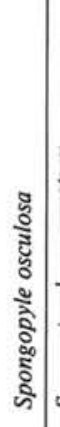 & 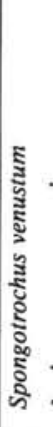 & 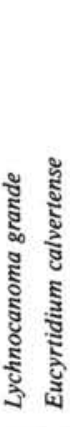 & 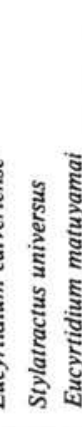 & 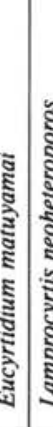 & 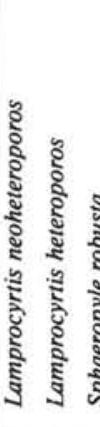 & 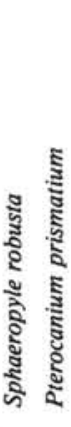 & 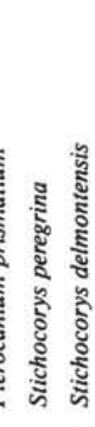 \\
\hline \multirow{3}{*}{ Pleistocene } & B. aquilonaris & $\begin{array}{l}1-1,10-11 \\
2-1,1-2 \\
3-1,1-2\end{array}$ & $\begin{array}{l}66 \\
19 \\
29\end{array}$ & \begin{tabular}{|c|} 
M-P \\
P \\
M \\
\end{tabular} & $\begin{array}{l}-\bar{R} \\
=-\end{array}$ & $\begin{array}{ll}\text { R } & \\
\text { F } & \\
\text { F } & \end{array}$ & $\begin{array}{ll}R & R \\
+ & R \\
R & R\end{array}$ & $\begin{array}{l}+ \\
+ \\
+\end{array}$ & $\begin{array}{l}+ \\
\mathbf{R} \\
+ \\
+\end{array}$ & $\begin{array}{l}+ \\
+ \\
+\end{array}$ & $\begin{array}{l}-\quad \mathrm{R} \\
-\quad+ \\
- \\
\end{array}$ & $\begin{array}{l}\bar{R} \\
\mathrm{R}\end{array}$ & $\begin{array}{l}\mathrm{R} \\
\mathrm{F} \\
\mathrm{F}\end{array}$ & $\begin{array}{l}\mathrm{R} \\
\mathrm{R} \\
\mathrm{R}\end{array}$ & $\begin{array}{l}-\mathrm{F} \\
-\mathrm{F} \\
+\mathrm{F}\end{array}$ & $\begin{array}{l}- \\
+ \\
\mathrm{R}\end{array}$ & $\begin{array}{l}R \\
R \\
F\end{array}$ & $\begin{array}{l}+ \\
\mathrm{R} \\
\mathrm{R}\end{array}$ & $\begin{array}{ll}+ & R \\
R & F \\
R & F\end{array}$ & $\begin{array}{l}R \\
R \\
R \\
R\end{array}$ & $\begin{array}{ll}R & R \\
R & + \\
R & R\end{array}$ & $\begin{array}{l}R \\
+ \\
F\end{array}$ & $\begin{array}{l}R \\
R \\
R\end{array}$ & $\begin{array}{l}\mathbf{R} \\
\mathbf{R} \\
\mathbf{R}\end{array}$ & $\begin{array}{l}\mathrm{F} \\
\mathrm{C} \\
\mathrm{F}\end{array}$ & $\begin{array}{l}-- \\
-\overline{-}\end{array}$ & $=$ & & - & & \\
\hline & S. universus & $\begin{array}{l}4-1,1-2 \\
5-1,10-12\end{array}$ & $\begin{array}{l}12 \\
37\end{array}$ & $\begin{array}{l}\mathrm{P} \\
\mathrm{M}\end{array}$ & $=-$ & $\begin{array}{ll}\mathrm{F} & \mathrm{F}\end{array}$ & $\begin{array}{rr}R & R \\
+\quad R\end{array}$ & $\bar{R}$ & $\begin{array}{l}\mathbf{R} \\
\mathrm{R}\end{array}$ & $\begin{array}{l}\mathbf{R} \\
\mathrm{R}\end{array}$ & $-\frac{-}{-}$ & ++ & $\begin{array}{l}\mathrm{F} \\
\mathrm{F}\end{array}$ & $\stackrel{+}{\mathrm{R}}$ & $\begin{array}{rl}R & F \\
+\quad R\end{array}$ & $\overline{\mathrm{R}}$ & $\begin{array}{l}\mathrm{F} \\
\mathrm{F}\end{array}$ & $\begin{array}{l}\mathrm{R} \\
\mathrm{R}\end{array}$ & $\begin{array}{ll}R & F \\
R & F\end{array}$ & $=\begin{array}{l}+ \\
R\end{array}$ & + & $\begin{array}{l}F \\
F\end{array}$ & $\begin{array}{l}\mathrm{R} \\
\mathrm{R}\end{array}$ & $\begin{array}{l}\mathrm{F} \\
\mathrm{F}\end{array}$ & $\begin{array}{l}\mathrm{C} \\
\mathrm{F}\end{array}$ & $\bar{R} \bar{R}$ & $\begin{array}{r}\mathrm{R} \\
+\end{array}$ & -- & $\overline{-}-$ & - & \\
\hline & E. matuyamai & $\begin{array}{l}6-1,12-14 \\
7-1,100-102 \\
8-1,30-32\end{array}$ & $\begin{array}{l}22 \\
41 \\
25\end{array}$ & $\begin{array}{c}M \\
\text { G-M } \\
M\end{array}$ & $\begin{array}{l} \pm \mathrm{R} \\
-\mathbf{-} \\
-\end{array}$ & $\begin{array}{ll}\mathbf{R} & \mathbf{1} \\
\mathrm{F} & \mathbf{1} \\
\mathbf{R} & \end{array}$ & $\begin{array}{ll}R & + \\
R & R \\
+ & +\end{array}$ & $\begin{array}{l}+ \\
\mathrm{R} \\
+\end{array}$ & $\begin{array}{l}+ \\
\mathrm{R} \\
\mathrm{R}\end{array}$ & $\begin{array}{l}+ \\
+ \\
+\end{array}$ & $\begin{array}{l}+ \\
\mathrm{R} \\
+\end{array}$ & $\begin{array}{l}+ \\
\mathrm{R} \\
-\end{array}$ & $\begin{array}{l}\mathrm{F} \\
\mathrm{R} \\
\mathrm{R}\end{array}$ & $\begin{array}{l}\mathrm{R} \\
\mathrm{R} \\
\mathrm{R}\end{array}$ & $\begin{array}{ll}\mathrm{R} & \mathrm{F} \\
+ & \mathrm{R} \\
\mathrm{R} & \mathrm{R}\end{array}$ & $\begin{array}{l}\mathrm{R} \\
\mathrm{R} \\
\mathrm{R}\end{array}$ & $\begin{array}{l}\mathrm{R} \\
+ \\
\mathrm{R}\end{array}$ & $\begin{array}{ll}\mathbf{R} & \\
\mathrm{R} & \\
\mathrm{R} & \\
\end{array}$ & $\begin{array}{ll}R & F \\
R & F \\
R & F \\
\end{array}$ & $\begin{array}{ll}F & + \\
F & R \\
F\end{array}$ & $\begin{array}{ll}+ & + \\
R & R \\
R & R \\
\end{array}$ & $\begin{array}{l}F \\
R \\
R\end{array}$ & $\begin{array}{l}\mathrm{R} \\
\mathrm{R} \\
\mathrm{R}\end{array}$ & $\begin{array}{l}\mathrm{R} \\
\mathrm{R} \\
\mathrm{R}\end{array}$ & $\begin{array}{l}\mathrm{C} \\
\mathrm{F} \\
\mathrm{C}\end{array}$ & $\begin{array}{l}-R \\
+\quad R \\
-\end{array}$ & $\begin{array}{l}+ \\
\mathrm{R} \\
+ \\
+\end{array}$ & \begin{tabular}{l|l}
+ & - \\
+ & + \\
$\mathrm{R}$ & $\mathbf{R}$ \\
\end{tabular} & $\begin{array}{lll}- & R & - \\
+ & R & R \\
R & + & R\end{array}$ & $\begin{array}{l}\bar{R}+ \\
R-\end{array}$ & - \\
\hline \multirow[b]{2}{*}{ Pliocene } & L. heteroporos & $8, \mathrm{CC}$ & 5 & $\mathrm{M}$ & - & $\mathrm{F} \mathrm{I}$ & R R & - & $\mathbf{R}$ & $\mathbf{R}$ & - & - & $\mathrm{R}$ & $\mathbf{R}$ & $R-$ & + & $\mathbf{R}$ & $\mathrm{R}$ & $+F$ & $F+$ & $+R$ & $F$ & $R$ & $R$ & $\mathrm{~F}$ & $\begin{array}{ll}R & R \\
\end{array}$ & $\mathrm{R}$ & -- & $--\mathrm{R}$ & $R-$ & --- \\
\hline & S. langii & $\begin{array}{l}10-1,10-11 \\
11-1,15-17 \\
12-1,15-17\end{array}$ & $\begin{array}{l}35 \\
50 \\
46\end{array}$ & $\begin{array}{c}M \\
\text { G-M } \\
\text { G } \\
\end{array}$ & & $\begin{array}{l}- \\
- \\
-\end{array}$ & $\begin{array}{l}-R \\
-E \\
--\end{array}$ & - & $\begin{array}{l}\mathrm{R} \\
- \\
-\end{array}$ & $\begin{array}{l}\mathrm{R} \\
\mathrm{R} \\
\mathrm{R}\end{array}$ & $\begin{array}{l}\mathrm{R} \\
- \\
-\end{array}$ & $\begin{array}{l}-\bar{R} \\
\mathrm{R}\end{array}$ & $\begin{array}{l}\mathrm{F} \\
\mathrm{R} \\
\mathrm{R}\end{array}$ & $\begin{array}{l}\mathrm{R} \\
\mathrm{R} \\
-\end{array}$ & $\begin{array}{ll}\mathrm{R} & - \\
\mathrm{R} & \mathrm{R} \\
\mathrm{R} & -\end{array}$ & $\begin{array}{l}R \\
+ \\
R\end{array}$ & $\begin{array}{l}F \\
R \\
R\end{array}$ & $\begin{array}{l}\mathrm{R} \\
\mathrm{R} \\
\mathrm{R}\end{array}$ & $\begin{array}{ll}R & F \\
+ & R \\
- & R\end{array}$ & $\begin{array}{ll}F & R \\
R & R \\
R & R\end{array}$ & $\begin{array}{ll}R & R \\
R & + \\
R & +\end{array}$ & $\begin{array}{r}F \\
F \\
-R\end{array}$ & $\begin{array}{l}- \\
+ \\
R\end{array}$ & $\begin{array}{l}\mathbf{R} \\
\mathbf{R} \\
\mathbf{R}\end{array}$ & $\begin{array}{l}\mathrm{F} \\
\mathrm{F} \\
\mathrm{C}\end{array}$ & $\begin{array}{ll}+ & R \\
- & + \\
- & R\end{array}$ & $\begin{array}{l}\mathrm{R} \\
\mathrm{R} \\
+\end{array}$ & $-\frac{-}{\mathrm{R}}$ & $\begin{array}{l}-\bar{R} \\
\bar{R}-\end{array}$ & $\begin{array}{ll}R^{R} & \\
+ & \\
& F\end{array}$ & $\begin{array}{l}R \\
\begin{array}{l}R \\
R \\
R\end{array}\end{array}$ \\
\hline $\begin{array}{c}\text { late } \\
\text { Miocene }\end{array}$ & S. peregrina & $\begin{array}{l}13-1,20-22 \\
14-1,80-82\end{array}$ & $\begin{array}{r}44 \\
6\end{array}$ & \begin{tabular}{|c|} 
M \\
$M-P$
\end{tabular} & & - & - & & - & $\overline{-}$ & & & $\begin{array}{l}\mathrm{R} \\
\mathrm{R}\end{array}$ & $\stackrel{+}{\mathrm{t}}$ & $\begin{array}{l}\mathrm{R}- \\
\mathrm{R}-\end{array}$ & ${ }_{R}^{+}$ & $\begin{array}{l}R \\
R\end{array}$ & $\begin{array}{l}\mathrm{R} \\
\mathrm{F}\end{array}$ & $\begin{array}{ll}\bar{F} & R \\
F\end{array}$ & \begin{tabular}{l|l}
$R$ & $R$ \\
$F$ & $R$
\end{tabular} & $\begin{array}{ll}R & R \\
R & R\end{array}$ & $\begin{array}{l}R \\
F\end{array}$ & + & $\begin{array}{l}\mathrm{R} \\
\mathrm{F}\end{array}$ & F & $\begin{array}{ll}\bar{R} & + \\
R\end{array}$ & $\begin{array}{l}R \\
R\end{array}$ & & $\begin{array}{l}-\mathrm{R} \\
- \\
-\end{array}$ & $\begin{array}{l}\mathrm{F} \\
\mathrm{R}\end{array}$ & $\begin{array}{l}R \\
+\end{array}$ \\
\hline
\end{tabular}

Note: Cores 15-20 are barren of radiolaria. See Procedures and Analyses for explanation of entries. 
Table 4. Abundances of some radiolarians from Holes 579 and 579A.

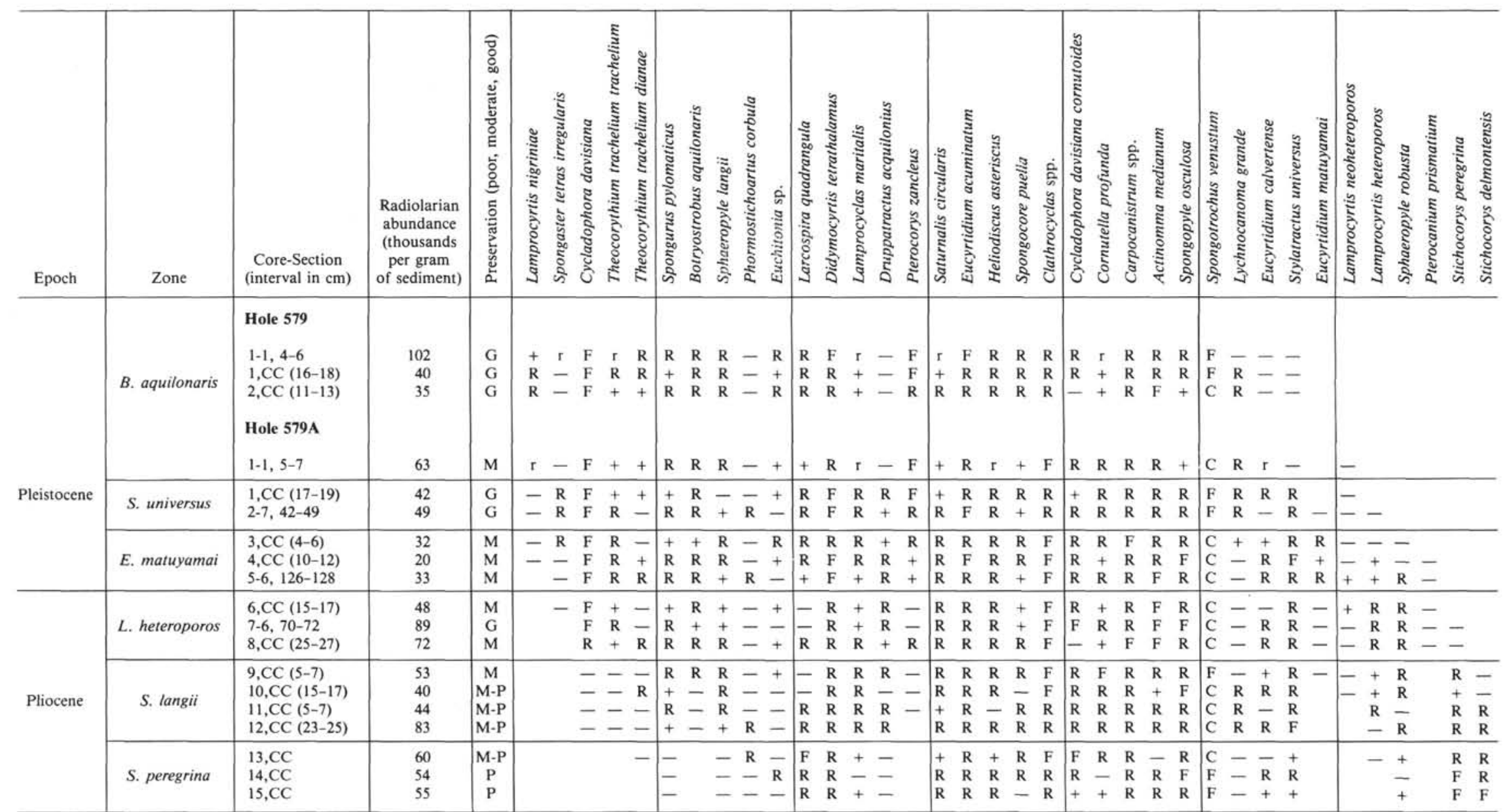

Note: See Procedures and Analyses for explanation of entries. 
Table 5. Abundances of some radiolarians from Hole 580 .

\begin{tabular}{|c|c|c|c|c|c|c|c|c|c|c|c|c|c|c|c|c|c|c|c|c|c|c|c|c|c|c|c|c|c|c|c|c|c|c|}
\hline Epoch & Zone & $\begin{array}{c}\text { Core-Section } \\
\text { (interval in } \mathrm{cm} \text { ) }\end{array}$ & $\begin{array}{c}\text { Radiolarian } \\
\text { abundance } \\
\text { (thousands } \\
\text { per gram } \\
\text { of sediment) }\end{array}$ & 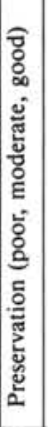 & 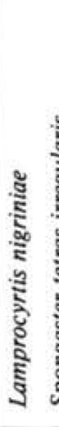 & 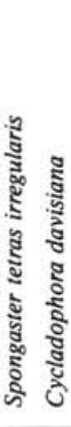 & 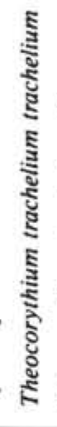 & 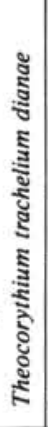 & 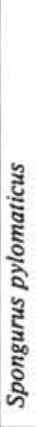 & 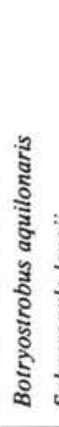 & 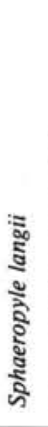 & 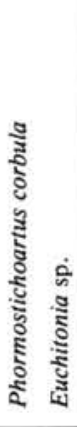 & & 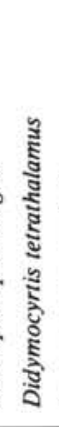 & 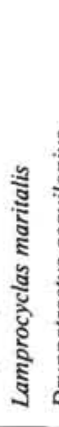 & 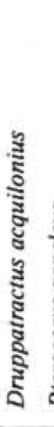 & 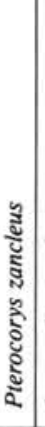 & 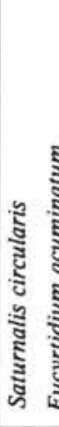 & 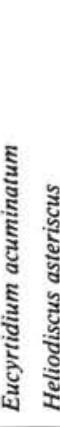 & 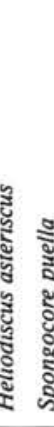 & 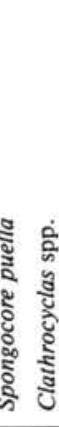 & & 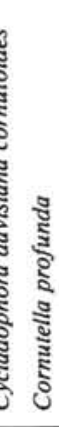 & 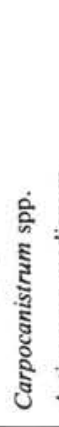 & 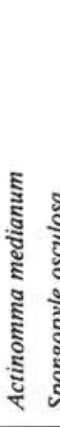 & 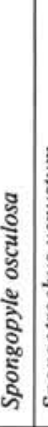 & 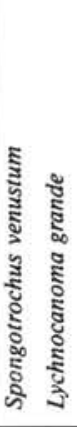 & 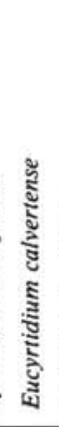 & 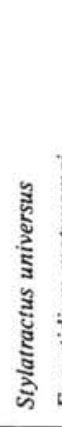 & 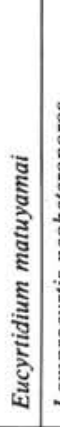 & 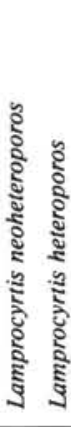 & 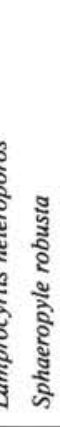 & 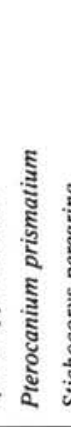 & 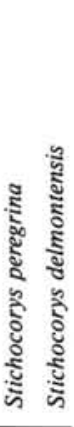 \\
\hline \multirow{3}{*}{ Pleistocene } & B. aquilonaris & $\begin{array}{l}1-1,3-5 \\
1, C C^{(5-7)} \\
2, C C(5-7) \\
3-6,105-107\end{array}$ & $\begin{array}{l}52 \\
20 \\
12 \\
18\end{array}$ & $\begin{array}{l}G \\
G \\
G \\
G\end{array}$ & $\begin{array}{l}- \\
- \\
\bar{R}\end{array}$ & $\begin{array}{ll}R & R \\
- & F \\
- & C \\
R & F\end{array}$ & $\begin{array}{l}+ \\
+ \\
+ \\
+\end{array}$ & $\begin{array}{l}+ \\
\mathbf{R} \\
+ \\
+\end{array}$ & $\begin{array}{l}\mathrm{R} \\
\mathrm{R} \\
+ \\
\mathrm{R}\end{array}$ & $\begin{array}{l}\mathrm{F} \\
\mathrm{R} \\
+ \\
+ \\
+\end{array}$ & $\begin{array}{l}+ \\
\mathrm{R} \\
+ \\
\mathrm{R}\end{array}$ & $\begin{array}{l}-\mathrm{R} \\
-\quad \mathrm{R} \\
-\quad+ \\
-\quad+\end{array}$ & $\overline{\mathrm{R}}$ & $\begin{array}{l}\text { R } \\
+ \\
+ \\
+\end{array}$ & $\begin{array}{l}- \\
- \\
- \\
-\end{array}$ & $\begin{array}{ll}- & 1 \\
- & 1 \\
- & 1 \\
R & 1\end{array}$ & $\begin{array}{l}\mathrm{F} \\
\mathrm{F} \\
\mathrm{F} \\
\mathrm{R}\end{array}$ & $\begin{array}{ll}+ & R \\
+ & R \\
+ & R \\
R & R\end{array}$ & $\begin{array}{ll}\mathrm{R} & \\
\mathrm{R} & \mathrm{R} \\
\mathrm{R} & \mathrm{Z} \\
\mathrm{R} & \mathrm{R}\end{array}$ & $\begin{array}{ll}- & R \\
R & + \\
& + \\
R & R \\
\end{array}$ & $\begin{array}{ll}\mathrm{R} & \mathrm{F} \\
+ & \mathrm{R} \\
\mathrm{R} & \mathrm{F} \\
\mathrm{R} & \mathrm{F} \\
\end{array}$ & & $\begin{array}{l}+ \\
R \\
R \\
R \\
R\end{array}$ & $\begin{array}{l}\mathrm{R} \\
\frac{-}{\mathrm{R}}\end{array}$ & $\begin{array}{ll}\mathrm{F} & + \\
\mathrm{R} & \mathrm{R} \\
\mathrm{R} & + \\
\mathrm{R} & \mathrm{R} \\
\end{array}$ & \begin{tabular}{l|l}
+ & \\
R & \\
+ & \\
R & \\
\end{tabular} & $\begin{array}{ll}\mathrm{C} & - \\
\mathrm{C} & \mathrm{R} \\
\mathrm{F} & \mathrm{F} \\
\mathrm{C} & \mathrm{F}\end{array}$ & $\begin{array}{l}- \\
- \\
-\end{array}$ & $\begin{array}{l}\bar{z} \\
\bar{z}\end{array}$ & & - & & & \\
\hline & S. universus & $\begin{array}{l}4, C C(20-22) \\
5, C C(30-32) \\
6, C C(26-28)\end{array}$ & $\begin{array}{l}21 \\
15 \\
23\end{array}$ & $\begin{array}{l}\text { G } \\
G \\
G\end{array}$ & $\begin{array}{l}\mathrm{R} \\
- \\
-\end{array}$ & $\begin{array}{ll}R & F \\
- & C \\
- & F\end{array}$ & $\begin{array}{l}- \\
-\end{array}$ & $\begin{array}{l}\vec{R} \\
R\end{array}$ & $\begin{array}{l}\mathbf{R} \\
+ \\
\mathbf{R}\end{array}$ & $\begin{array}{l}\mathrm{R} \\
\mathrm{R} \\
-\end{array}$ & $\begin{array}{l}\mathrm{R} \\
\mathrm{R} \\
\mathrm{R}\end{array}$ & $\begin{array}{l}-\mathrm{R} \\
-\underline{-}\end{array}$ & $\frac{\mathrm{R}}{\mathrm{R}}$ & $\begin{array}{l}F \\
R \\
+\end{array}$ & $\begin{array}{l}-1 \\
-1 \\
-1\end{array}$ & $\begin{array}{ll}\mathrm{R} & \\
\mathrm{R} & \mathrm{1} \\
\mathrm{R} & \mathrm{I}\end{array}$ & $\begin{array}{l}\mathrm{F} \\
\mathrm{F} \\
\mathrm{F}\end{array}$ & $\begin{array}{ll}- & R \\
\bar{R} & R \\
R & R\end{array}$ & $\begin{array}{ll}\mathrm{R} & \mathrm{R} \\
\mathrm{R} & \mathrm{R} \\
\mathrm{R} & \mathrm{R}\end{array}$ & $\begin{array}{ll}R & R \\
R & R \\
R & R\end{array}$ & $\begin{array}{ll}\mathrm{R} & \mathrm{F} \\
- & \mathrm{F} \\
\mathrm{R} & \mathrm{F}\end{array}$ & $\begin{array}{l}R \\
R \\
F\end{array}$ & $\begin{array}{l}- \\
+ \\
R\end{array}$ & $\begin{array}{l}\mathrm{R} \\
\mathrm{R} \\
\mathrm{R}\end{array}$ & $\begin{array}{ll}F & F \\
R & F \\
R & F\end{array}$ & $\begin{array}{l}F \\
F \\
F\end{array}$ & $\begin{array}{ll}\mathrm{F} & \mathrm{F} \\
\mathrm{C} & + \\
\mathrm{F} & \mathrm{R}\end{array}$ & $\frac{\mathrm{R}}{-}$ & $\begin{array}{l}+ \\
\mathrm{R} \\
\mathrm{R}\end{array}$ & $\begin{array}{ll}- & - \\
- & \\
-\end{array}$ & $\begin{array}{l}-\overline{-} \\
\bar{R}\end{array}$ & - & & \\
\hline & E. matuyamai & $\begin{array}{l}7-6,5-7 \\
8, C C \quad(30-32) \\
9, C C(35-37) \\
10, C C(35-37) \\
\end{array}$ & $\begin{array}{l}12 \\
16 \\
32 \\
39 \\
\end{array}$ & \begin{tabular}{l|}
$\mathrm{M}$ \\
$\mathrm{G}$ \\
$\mathrm{G}$ \\
$\mathrm{G}$ \\
\end{tabular} & $\overline{-}$ & $\begin{array}{r}-\mathrm{C} \\
-\mathrm{F} \\
\mathrm{F} \\
\mathrm{F}\end{array}$ & $\begin{array}{l}\mathrm{R} \\
- \\
+\end{array}$ & $\begin{array}{l}- \\
z \\
- \\
- \\
\end{array}$ & $\begin{array}{l}\mathrm{R} \\
\mathrm{R} \\
+ \\
\mathrm{R} \\
\end{array}$ & $\begin{array}{l}- \\
+ \\
+ \\
-\end{array}$ & $\begin{array}{l}\mathrm{R} \\
\mathrm{R} \\
+ \\
+ \\
\end{array}$ & $\begin{array}{l}-\frac{-}{-} \\
\overline{-} \\
+-\end{array}$ & $\begin{array}{l}- \\
- \\
-\end{array}$ & $\begin{array}{l}R \\
R \\
+ \\
R \\
\end{array}$ & $\begin{array}{l}+ \\
- \\
+ \\
+ \\
+ \\
\end{array}$ & $\begin{array}{ll} & \\
R & \\
R & \\
R & \\
\end{array}$ & $\begin{array}{l}+ \\
\\
\mathrm{R} \\
-\end{array}$ & $\begin{array}{ll}R & R \\
R & R \\
R & R \\
R & R \\
\end{array}$ & $\begin{array}{ll}\mathrm{R} & \\
\mathrm{R} & \mathrm{R} \\
\mathrm{R} & + \\
\mathrm{R} & \mathrm{R} \\
\end{array}$ & $\begin{array}{ll} & - \\
\mathrm{R} & = \\
+ & = \\
\mathrm{R} & \mathrm{R} \\
\end{array}$ & $\begin{array}{ll}- & \mathrm{R} \\
- & \mathrm{R} \\
- & \mathrm{F} \\
\mathrm{R} & \mathrm{R} \\
\end{array}$ & $\begin{array}{l}R \\
R \\
R \\
R\end{array}$ & $\begin{array}{l}- \\
+ \\
R \\
R\end{array}$ & $\begin{array}{l}+ \\
\overline{\mathrm{R}} \\
+ \\
\end{array}$ & $\begin{array}{ll}R & F \\
R & F \\
R & R \\
R & F \\
\end{array}$ & \begin{tabular}{l|l}
$\mathrm{F}$ & \\
$\mathrm{F}$ & \\
$\mathrm{R}$ & \\
$\mathrm{F}$ & \\
\end{tabular} & $\begin{array}{ll}\mathrm{C} & \mathrm{R} \\
\mathrm{C} & + \\
\mathrm{C} & \mathrm{R} \\
\mathrm{C} & \mathrm{R} \\
\end{array}$ & $\begin{array}{l}+ \\
+ \\
+ \\
+ \\
\end{array}$ & $\begin{array}{l}\mathrm{R} \\
\mathrm{R} \\
\mathrm{F} \\
\mathrm{R} \\
\end{array}$ & \begin{tabular}{l|l}
$\mathbf{F}$ & - \\
$\mathbf{R}$ & - \\
+ & + \\
+ & $\mathbf{R}$ \\
\end{tabular} & $\begin{array}{ll}- & R \\
- & \frac{R}{R} \\
& R \\
\end{array}$ & $\begin{array}{l}- \\
+ \\
R \\
+\end{array}$ & + & \\
\hline \multirow[t]{2}{*}{ Pliocene } & L. heteroporos & $\begin{array}{l}11, C C(20-22) \\
12, C C(10-12) \\
13, C C(5-7) \\
14, C C(5-7) \\
15, C C(5-7)\end{array}$ & $\begin{array}{l}34 \\
40 \\
61 \\
37 \\
47\end{array}$ & $\begin{array}{l}G \\
G \\
G \\
G \\
G\end{array}$ & & $\begin{array}{l}\mathrm{F} \\
\mathrm{F} \\
\mathrm{F} \\
\mathrm{F} \\
\mathrm{R}\end{array}$ & $\begin{array}{l}- \\
- \\
-\end{array}$ & & $\begin{array}{l}+ \\
+ \\
\mathrm{R} \\
+ \\
\mathrm{R}\end{array}$ & $\begin{array}{l}+ \\
\mathrm{R} \\
\overline{\mathrm{R}}\end{array}$ & $\begin{array}{l}\mathrm{R} \\
+ \\
\mathrm{r} \\
+ \\
\mathrm{R}\end{array}$ & $\begin{array}{ll}\bar{R} & - \\
\mathrm{R} & \mathrm{r} \\
\mathrm{R} & \mathrm{R} \\
- & -\end{array}$ & & $\begin{array}{l}+ \\
R \\
R \\
R \\
+\end{array}$ & $\begin{array}{l}-1 \\
- \\
- \\
\bar{R}\end{array}$ & $\begin{array}{l}\mathrm{R} \\
\mathrm{R} \\
+ \\
\mathrm{R} \\
\mathrm{R} \\
+\end{array}$ & $\begin{array}{l}- \\
- \\
\bar{R}\end{array}$ & $\begin{array}{ll}- & R \\
- & R \\
r & R \\
R & R \\
R & R \\
R & R\end{array}$ & $\begin{array}{ll}\mathrm{R} & + \\
\mathrm{R} & \mathrm{R} \\
\mathrm{R} & + \\
\mathrm{R} & + \\
\mathrm{R} & \mathrm{R}\end{array}$ & $\begin{array}{ll}+ & + \\
R & + \\
+ & + \\
+ & + \\
\text { R } & \text { R }\end{array}$ & $\begin{array}{ll}+ & R \\
+ & F \\
+ & R \\
+ & R \\
R & F\end{array}$ & $\begin{array}{l}\mathrm{R} \\
\mathrm{F} \\
\mathrm{F} \\
\mathrm{F} \\
\mathrm{F}\end{array}$ & $\begin{array}{l}R \\
R \\
F \\
R \\
R\end{array}$ & $\begin{array}{l}\mathrm{R} \\
\mathrm{R} \\
\mathrm{R} \\
\mathrm{R} \\
\mathrm{R}\end{array}$ & $\begin{array}{ll}\mathrm{F} & \mathrm{F} \\
\mathrm{F} & \mathrm{F} \\
\mathrm{R} & \mathrm{R} \\
\mathrm{R} & \mathrm{F} \\
- & \mathrm{F}\end{array}$ & $\begin{array}{l}F \\
F \\
R \\
F \\
F\end{array}$ & $\begin{array}{ll}\mathrm{C} & \mathrm{R} \\
\mathrm{F} & + \\
\mathrm{F} & \mathrm{R} \\
\mathrm{C} & - \\
\mathrm{C} & +\end{array}$ & $\begin{array}{l}+ \\
+ \\
+ \\
+ \\
+\end{array}$ & $\begin{array}{l}\mathrm{R} \\
+ \\
\mathrm{R} \\
\mathrm{R} \\
\mathrm{R}\end{array}$ & $\begin{array}{ll}- & 1 \\
- & - \\
- & -\end{array}$ & $\begin{array}{ll}\mathrm{R} & \mathrm{F} \\
- & \mathrm{R} \\
- & \mathrm{R} \\
- & \mathrm{R} \\
- & \mathrm{R}\end{array}$ & $\begin{array}{l}R \\
R \\
r \\
R \\
R\end{array}$ & $\overline{-}$ & - \\
\hline & S. langii & $\begin{array}{l}16-6,120-122 \\
17, C C(13-15)\end{array}$ & $\begin{array}{l}44 \\
40\end{array}$ & $\begin{array}{l}G \\
G\end{array}$ & & - & & & & $\stackrel{R}{-}$ & $\overline{\mathrm{R}}$ & $-\underline{-}$ & & $\begin{array}{l}\mathrm{R} \\
\mathrm{R}\end{array}$ & $\bar{R}$ & $\begin{array}{l}\mathrm{R} \\
\mathrm{R}\end{array}$ & - & $\begin{array}{ll}\mathrm{R} & \mathrm{R} \\
\mathrm{R} & -\end{array}$ & $\begin{array}{ll}\mathbf{R} & \mathbf{R} \\
- & +\end{array}$ & $\begin{array}{l}R+ \\
++\end{array}$ & $\begin{array}{l}+\mathrm{R} \\
+\mathrm{F}\end{array}$ & & $\begin{array}{l}R \\
R \\
R\end{array}$ & $\begin{array}{l}\mathbf{R} \\
\mathbf{R}\end{array}$ & $\begin{array}{ll}+ & F \\
R & F\end{array}$ & $\begin{array}{l}\mathrm{F} \\
\mathrm{F}\end{array}$ & $\begin{array}{ll}\mathrm{F} & \mathrm{R} \\
\mathrm{F} & -\end{array}$ & + & $\begin{array}{l}R \\
R\end{array}$ & & $\begin{array}{l}R \\
R\end{array}$ & $\begin{array}{l}R \\
R\end{array}$ & & $\begin{array}{l}\mathrm{R}- \\
\mathrm{R}-\end{array}$ \\
\hline
\end{tabular}

Note: See Procedures and Analyses for explanation of entries. 
the upper limit of Stylatractus universus occurs between Samples 580-4-1, 65-67 cm and 580-4-1, 95-97 cm, with the upper limit of Eucyrtidium matuyamai occurring between Samples 580-7-2, 5-7 cm and 580-7-2, 35-37 cm. These two biostratigraphic datum levels define the top and bottom of the $S$. universus Zone. The first occurrence of $E$. matuyamai is found in the uppermost sample from Core 11 (Sample 580-11-1, 10-11 cm, based on more extensive sampling than shown in Table 5), indicating that the early Pleistocene E. matuyamai Zone extends from Core 7 through Core 10. Fauna characteristic of the late Pliocene Lamprocyrtis heteroporos Zone is present in Cores 11 through 15, with the bottom of this zone coinciding with the latest occurrence of Stichocorys peregrina located between Samples 580-15,CC $(5-7 \mathrm{~cm})$ and $580-16-6,120-122 \mathrm{~cm}$. The radiolarians in sediments from Cores 16 and 17 are representative of the Sphaeropyle langii Zone. The absence of Stichocorys delmontensis from the oldest sediment recovered from Hole 580 in conjunction with the magnetostratigraphy indicates that drilling terminated above the biostratigraphic and paleomagnetic boundary between early and late Pliocene.

\section{Site 581}

Site 581 was selected to sample siliceous sediments in a high-deposition subarctic setting. Radiolarians with various states of preservation are preserved in Neogene and Quaternary siliceous clay sediments from this site (Table 6).

The echo character of the seismic profiles from this site suggests that the uppermost sediment may represent a debris-flow deposit. Although a mixed fauna was not detected in Core 1 collected at the mudline, the radiolarians present throughout this core are characteristic of the Stylatractus universus Zone, indicating that either latest Pleistocene sediments containing fauna of the Botryostrobus aquilonaris Zone were not deposited at this site or these younger sediments were reworked into older late Pleistocene sediments as a result of some bottom-current process. Because of the overall high Neogene and Quaternary siliceous sedimentation rates and the nature of the seismic profiles at this site, the latter hypothesis appears more plausible. Because of time constraints no sediment was recovered from approximately the next $181 \mathrm{~m}$ of sediment. Continuous rotary drilling commenced at a sub-bottom depth of $181.5 \mathrm{~m}$. Fauna in the uppermost sediments from Core 2 (Sample $581-2-1,10-12 \mathrm{~cm}$ ) is representative of the early Pliocene marked by the presence of Stichocorys delmontensis and Sphaeropyle langii. Likewise, the radiolarians present in the remainder of Core 2 are characteristic of the early Pliocene S. langii Zone. The sediment sequence from Core 3 through the top of Core 5 contains a fauna characteristic of the Stichocorys peregrina Zone. The fauna in the remainder of Core 5 through the top of Core 6 is representative of the Didymocyrtis penultima Zone. The Didymocyrtis antepenultima Zone extends throughout most of Core 6 into the upper portion of Core 7, with the radiolarians in the remainder of Core 7 into the top of Core 8 indicative of the Diartus petterssoni Zone. The fauna in most of Core 8 and in that portion of Core 9 where radiolar- ians are present is characteristic of the Dorcadospyris alata Zone.

\section{STRATIGRAPHIC AND GEOGRAPHIC RADIOLARIAN RANGES}

A complete stratigraphic range chart for selected Neogene and Quaternary radiolarians in sediments from Sites 577 through 581 is shown in Figure 2. The high recovery rate and quality of radiolarian preservation at the various sites drilled during Leg 86 make it possible to examine various biostratigraphic datum levels across a broad latitudinal band $\left(\sim 12^{\circ}\right)$. These analyses are summarized as follows:

1. The last abundant appearance of Lychnocanoma grande occurs near the top of the Botryostrobus aquilonaris Zone. Analyses of the correlation of this datum level with the relative abundance pattern of Cycladophora davisiana at closely spaced sample intervals at Sites 579 and 580 (not all shown in Tables 4 and 5) shows that this event is synchronous in this region.

2. Correlation of the latest occurrence of Druppatractus acquilonius with the relative abundance pattern of $C$. davisiana at closely spaced sample intervals at Sites 579 and 580 (not all shown in Tables 4 and 5) indicates that this datum is synchronous in this region, occurring just above the base of the $B$. aquilonaris Zone.

3. Based on correlation with the relative abundance pattern of C. davisiana and specific ash layers in sediments from Sites 579 and 580 (not all samples shown in Tables 4 and 5), the latest occurrence of Stylatractus universus appears to be synchronous throughout the region, confirming the results reported by Hays and Shackleton (1976) and Morley and Shackleton (1978).

4. Examination at closely spaced sample intervals at Sites 579 and 580 (not all shown in Tables 4 and 5) indicates that the latest occurrence of Eucyrtidium matuyamai occurs just above the base of the Jaramillo Subchronozone. Although samples were not taken at close intervals at Sites 577 or 578 , the preliminary findings from these sites are in agreement with those from Sites 579 and 580 , indicating that this event is synchronous throughout the northwest Pacific.

5. Because of the scarcity of E. matuyamai and close morphological characteristics to $E$. calvertense at the time of its first appearance, it is difficult to define the first occurrence of $E$. matuyamai as precisely as the previous four biostratigraphic markers. Detailed analyses at Sites 579 and 580 (not all samples shown in Tables 4 and 5), however, indicate that this datum level occurs within the Olduvai Subchronozone, which is in agreement with the previous findings of Hays (1970) and Kellogg and Hays (1975). Preliminary analyses of this species throughout its range indicate that the mean shell width of this assemblage is smallest in the southernmost site (Site 578) and largest in the northernmost site (Site 580). Hays (1970) first suggested that such a trend existed from his analyses of samples from several lower-sedimentationrate cores in the North Pacific.

6. Pterocanium prismatium was present in samples from Holes 577, 578, and 580. The latest occurrence of $P$. prismatium in Hole 577 is located between Samples $577-3-4,67-68 \mathrm{~cm}$ and 577-3,CC. The Pliocene/Pleisto- 
Table 6. Abundances of some radiolarians from Hole 581 .

\begin{tabular}{|c|c|c|c|c|c|c|c|c|c|c|c|c|c|c|c|c|c|c|c|c|c|c|c|c|c|c|c|c|}
\hline Epoch & Zone & $\begin{array}{l}\text { Core-Section } \\
\text { (interval in cm) }\end{array}$ & $\begin{array}{l}\text { Radiolarian } \\
\text { abundance } \\
\text { (thousands } \\
\text { per gram } \\
\text { of sediment) }\end{array}$ & 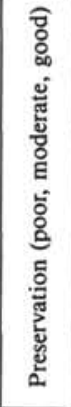 & 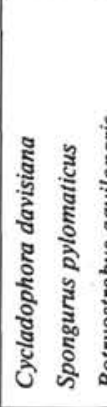 & 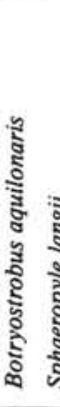 & 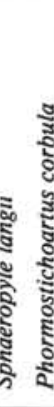 & 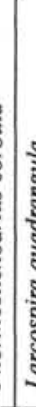 & 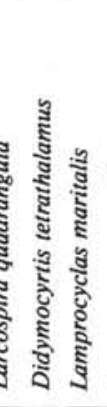 & 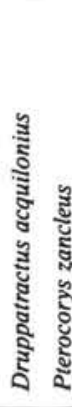 & 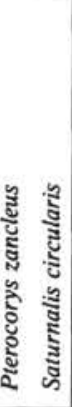 & 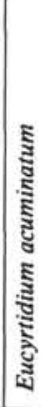 & 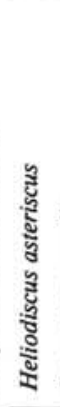 & 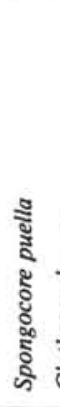 & 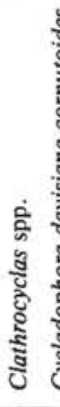 & & 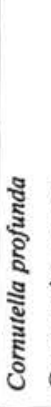 & 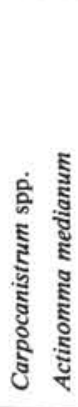 & 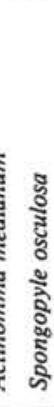 & 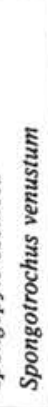 & 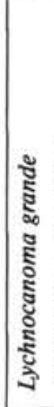 & 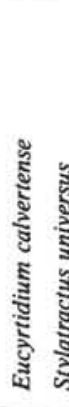 & 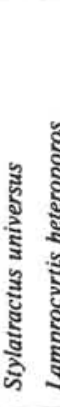 & 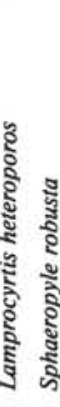 & 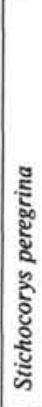 & 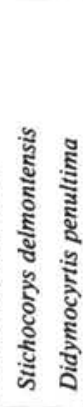 & 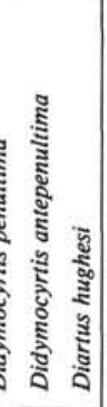 & 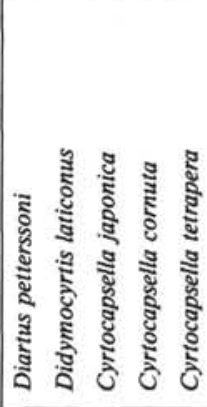 \\
\hline Pleistocene & S. universus & $\begin{array}{l}1-1,9-10 \\
1, \mathrm{CC}\end{array}$ & $\begin{array}{l}96 \\
43 \\
\end{array}$ & $\begin{array}{l}\text { G } \\
\mathbf{G}\end{array}$ & $\begin{array}{lll}\mathbf{F} & \mathbf{F} & 1 \\
\mathbf{C} & \mathbf{R} & 1 \\
\end{array}$ & $\begin{array}{ll}F & R \\
F & R \\
\end{array}$ & $\begin{array}{ll}\mathrm{R} & - \\
\mathrm{R} & \mathrm{R} \\
\end{array}$ & R & $\begin{array}{l}+\quad R \\
R+\end{array}$ & $\begin{array}{r}+\mathrm{F} \\
+\mathrm{F} \\
\end{array}$ & $\begin{array}{l}\mathrm{F}- \\
\mathrm{F}-\end{array}$ & $\begin{array}{l}\mathrm{R} \\
\mathrm{R} \\
\end{array}$ & $\overline{-}$ & $\begin{array}{l}\mathrm{R} \\
\mathrm{R} \\
\end{array}$ & $\begin{array}{ll}\mathrm{F} & \mathrm{F} \\
\mathrm{F} & \mathrm{F} \\
\end{array}$ & & $\begin{array}{l}\mathbf{R} \\
\mathbf{R} \\
\end{array}$ & $\begin{array}{r}R R \\
-\quad R \\
\end{array}$ & $\begin{array}{l}R \\
R\end{array}$ & $\begin{array}{l}\mathrm{F} \\
\mathrm{F}\end{array}$ & $\begin{array}{l}\mathrm{F} \\
\mathrm{F} \\
\end{array}$ & $\begin{array}{l}-\mathrm{R} \\
-\mathrm{R} \\
\end{array}$ & $\begin{array}{l}\mathbf{R}- \\
\mathbf{R}-\end{array}$ & - & & & & \\
\hline Pliocene & S. langii & $\begin{array}{l}2-1,10-12 \\
2, \mathrm{CC}\end{array}$ & $\begin{array}{l}36 \\
56\end{array}$ & $\begin{array}{c}\text { G } \\
\text { G-M }\end{array}$ & $= \pm$ & $\begin{array}{l}+R \\
-\quad+\end{array}$ & $\begin{array}{l}\mathrm{R}- \\
+-\end{array}$ & - & $\begin{aligned} & R \\
&-+ \\
&\end{aligned}$ & $\begin{array}{l}\mathbf{R}+ \\
\mathbf{R}\end{array}$ & $\pm R$ & & $\begin{array}{l}\mathbf{R} \\
\mathrm{R} \\
\end{array}$ & $\begin{array}{ll}\mathbf{R} & 1 \\
\mathbf{R} & \\
\end{array}$ & $\begin{array}{ll}\mathrm{R} & \mathrm{R} \\
\mathrm{R} & \mathrm{R} \\
\end{array}$ & \begin{tabular}{l|l}
$\mathbf{R}$ & $\mathbf{1}$ \\
$\mathbf{R}$ & $\mathbf{I}$ \\
\end{tabular} & & $\begin{array}{l}-R \\
-\quad+\end{array}$ & $\begin{array}{l}\mathrm{R} \\
\mathrm{R}\end{array}$ & $\begin{array}{l}\mathrm{C} \\
\mathrm{C}\end{array}$ & & $\begin{array}{r}-\mathrm{F} \\
+\quad \mathrm{F} \\
\end{array}$ & $\begin{array}{ll}\mathrm{F} & \mathrm{F} \\
\mathrm{F} & \mathrm{R} \\
\end{array}$ & $\begin{array}{ll}\mathbf{F} & \mathbf{R} \\
\mathrm{R} & + \\
\end{array}$ & & $\begin{array}{l}\mathrm{F} \\
\mathrm{R}\end{array}$ & & \\
\hline \multirow{3}{*}{$\begin{array}{c}\text { Late } \\
\text { Miocene }\end{array}$} & S. peregrina & $\begin{array}{l}3, \mathrm{CC} \\
5-1,110-112\end{array}$ & $\begin{array}{l}62 \\
85\end{array}$ & \begin{tabular}{|l|} 
M-P \\
G-M
\end{tabular} & - & - & - $\mathrm{R}$ & & $\begin{array}{l}\mathrm{R}- \\
-\end{array}$ & $\begin{aligned} \mathrm{R} R \\
+\quad \mathrm{R} \\
\end{aligned}$ & $\begin{array}{|ll|}R & R \\
R & - \\
\end{array}$ & R & $\begin{array}{l}\mathrm{R} \\
\mathrm{R} \\
\end{array}$ & $\begin{array}{l}\mathbf{R} \\
+\quad 1 \\
\end{array}$ & $\begin{array}{ll}\mathrm{F} & \mathrm{R} \\
\mathrm{R} & \mathrm{R} \\
\end{array}$ & & & $\begin{array}{ll}\bar{R} & R \\
R & R \\
\end{array}$ & $\begin{array}{l}R \\
R\end{array}$ & $\begin{array}{l}\mathrm{C} \\
\mathrm{F}\end{array}$ & \begin{tabular}{|l|}
$\mathbf{R}$ \\
$\mathbf{R}$ \\
\end{tabular} & $\begin{array}{l}-R \\
-R \\
\end{array}$ & $\begin{array}{l}\mathbf{R}- \\
\mathbf{R}-\end{array}$ & $-\bar{R}$ & & $\begin{array}{l}\mathrm{R}- \\
\mathrm{F}-\end{array}$ & - & \\
\hline & D. penultima & $6-1,70-72$ & 150 & G-M & & & - & & - & $-\mathrm{R}$ & $\mathbf{R}+$ & R & + & -1 & R R & $\mathbf{R}$ & $\mathbf{R}$ & $\begin{array}{ll}R & R \\
\end{array}$ & $\mathrm{R}$ & $\mathrm{F}$ & + & $+R$ & $\mathbf{R}$ & + & & $\mathrm{F} \quad \mathrm{R}$ & -- & \\
\hline & D. antepenultima & $7-1,60-62$ & 126 & G-M & & & - & & & - & -- & R & $\mathrm{R}$ & R 1 & $R \quad R$ & $\mathbf{R}$ & $R$ & $\begin{array}{ll}\mathrm{R} & \mathrm{R} \\
\end{array}$ & $R$ & $\mathrm{C}$ & - & $-F$ & $\mathrm{~F}$ & $\mathbf{R}$ & $\mathrm{R}$ & $\mathrm{F}-$ & $\begin{array}{ll}-R & R \\
\end{array}$ & --- \\
\hline \multirow{2}{*}{$\begin{array}{l}\text { Mid } \\
\text { Miocene }\end{array}$} & D. petterssoni & $8-1,70-72$ & 134 & M-P & & & & & & & -- & & $\mathbf{R}$ & +1 & R $\mathrm{R}$ & $\mathbf{R}$ & $\mathbf{R}$ & $\mathrm{R}-$ & $F$ & $\mathrm{~F}$ & $R$ & $+\mathrm{R}$ & $\mathbf{R}$ & + & & $\mathrm{R}-$ & --+ & R R F -- \\
\hline & D. alata & $9-1,40-42$ & 15 & $P$ & & & & & & & & - & - & - & $-R$ & $\mathbf{R}$ & $R$ & R $\quad R$ & $\mathrm{~F}$ & $\mathrm{~F}$ & F & $-F$ & $\mathrm{~F}$ & $\mathbf{R}$ & & + & -- & $-\begin{array}{lll}\mathrm{R} & \mathrm{F} & \mathrm{C}\end{array}$ \\
\hline
\end{tabular}

Note: Cores 10-11 are barren of radiolaria. See Procedures and Analyses for explanation of entries. 


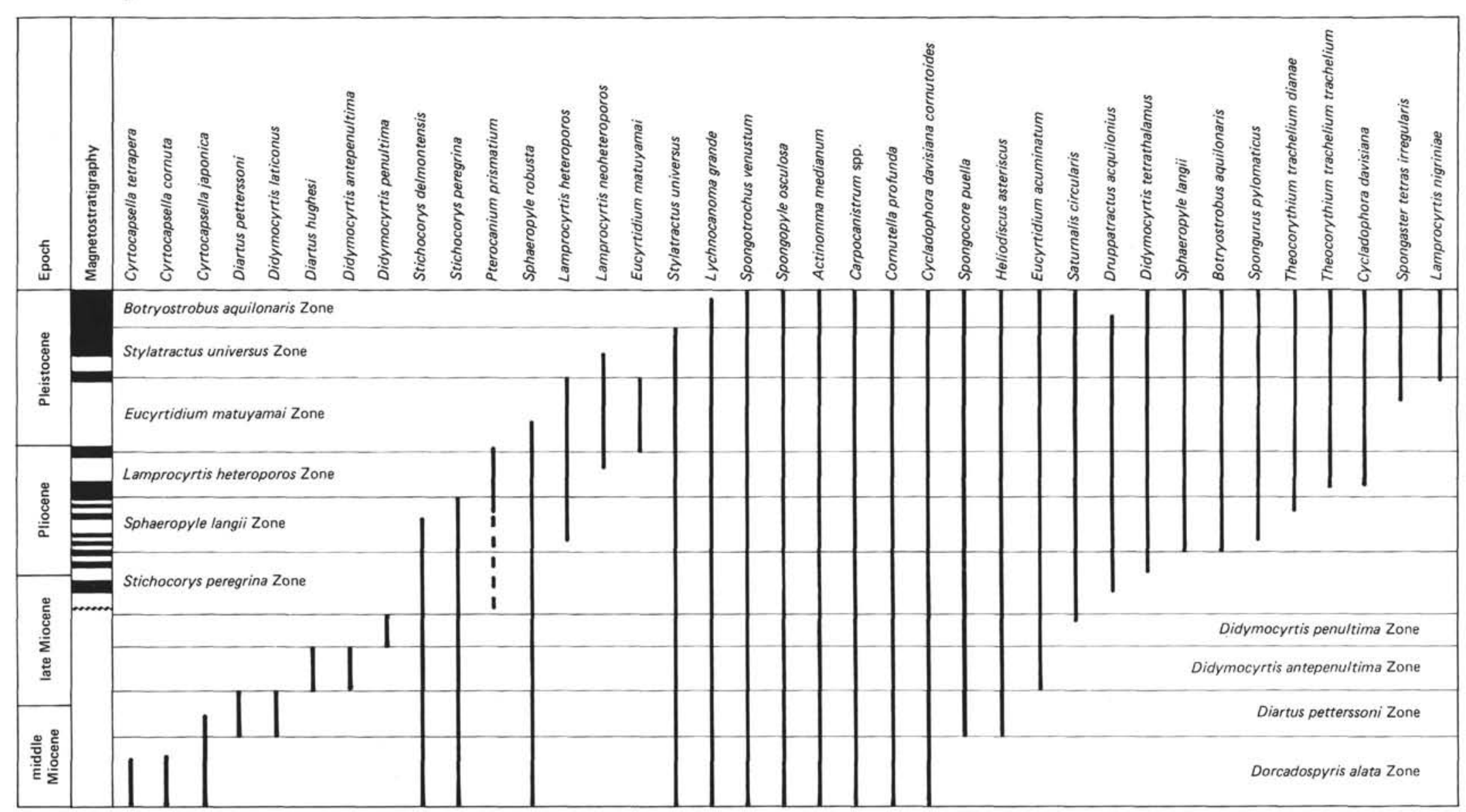

Figure 2. Range chart for Neogene and Quaternary radiolarians from DSDP Leg 86 sites. 
cene boundary based on magnetostratigraphy occurs in the middle of Core 3. At Site 578, the latest occurrence of $P$. prismatium is positioned less than a meter above the top of the Olduvai Subchronozone (Pliocene/Pleistocene boundary). This biostratigraphic marker occurs between Samples 580-9,CC (35-37 cm) and 580-10,CC $(35-37 \mathrm{~cm})$ in sediments from Hole 580 , with the Olduvai Subchronozone spanning the lower $6 \mathrm{~m}$ of Core 10 through the top meter of Core 11. These data show that $P$. prismatium ranges as far north as $42^{\circ} \mathrm{N}$ latitude in the Pacific and that although very rare in abundance, its upper limit occurs near the Pliocene/Pleistocene boundary based on magnetostratigraphy.

7. At most sites drilled on Leg 86 , the latest occurrence of Lamprocyrtis heteroporos was recorded near the top of the early Pleistocene Eucyrtidium matuyamai Zone.

8 . Based on examination of samples at closely spaced intervals at Sites 579 and 580 (not all shown in Tables 4 and 5), the first occurrence of Cycladophora davisiana occurs in the upper Gauss Chronozone near the base of the Lamprocyrtis heteroporos Zone approximately 2.7 m.y. ago.

9. Preliminary analyses indicate that Lamprocyrtis neoheteroporos ranges from the upper third of the $L$. heteroporos Zone through the lower two-fifths of the Stylatractus universus Zone. A similar range for this species in sediments from this region was reported by Foreman (1975).

10. At the sites where Theocorythium trachelium trachelium was present in high enough abundances (Sites $577,578,579$ ), the first occurrence of this species was detected at or near the base of the L. heteroporos Zone, which is in agreement with analyses reported by Foreman (1975).

11. The latest occurrence of Stichocorys peregrina was well documented in sediment sequences from all sites drilled during Leg 86 except Site 576 where radiolarians were not present in sediments older than Pleistocene. This event serves as an easily recognizable boundary between the bottom of the Lamprocyrtis heteroporos Zone and the top of the Sphaeropyle langii Zone throughout the northwest Pacific.

12. The latest occurrence of Stichocorys delmontensis coincides with both the Gilbert/Gauss magnetic polarity boundary and the early/late Pliocene boundary. This event is easily identified in samples from sites drilled on Leg 86 which contained siliceous sediments of this age (Sites 577, 578, 579).

13. The first occurrence of Lamprocyrtis heteroporos occurs near the base of the Sphaeropyle langii Zone.

14. Although the first occurrence of Sphaeropyle langii as registered in sediments from Sites 577, 578, 579, and 581 assisted in defining the top of the Stichocorys peregrina Zone, it was difficult at times to distinguish this species from others, such as Sphaeropyle robusta. The low abundance of this species in some samples made it difficult to identify the boundary between the Stichocorys peregrina Zone and the Sphaeropyle langii Zone.

15. At Site 581, the range of Didymocyrtis penulti$m a$ was used to delineate the $D$. penultima Zone.

16. The range of $D$. antepenultima was used to define the $D$. antepenultima Zone at Site 581 .
17. At Site 581, the ranges of Diartus petterssoni and Didymocyrtis laticonus were used to define the Diartus petterssoni Zone.

18. Preliminary analyses of samples from Site 581 indicate that the latest occurrence of Cyrtocapsella japonica occurred somewhere within the Diartus petterssoni Zone. This agrees with results from DSDP sites near Japan reported by Sakai (1980).

19. Results from Site 581 agree with that reported by Sakai (1980), placing the latest occurrence of C. tetrape$r a$ and $C$. cornuta in the upper half of the Dorcadospyris alata Zone.

\section{PALEOCEANOGRAPHY}

One of the major objectives of Leg 86 was to determine whether the initiation of significant biosiliceous accumulation in the Neogene was synchronous or transitional at the four sites roughly aligned along a northsouth transect (Sites 578, 579, 580, and 581) in the northwest Pacific. Although the hydraulic piston core did not penetrate deep enough to recover sediments recording this event at Sites 579 and 580, the onset of biosiliceous sedimentation in the northwest Pacific was recovered in sediments at the southernmost (Site 578) and northernmost (Site 581) sites. At Site 578, biosiliceous sedimentation commenced in the late Miiocene with earliest radiolarian fauna characteristic of the Stichocorys peregrina Zone, whereas at Site 581, biosiliceous sedimentation began in the middle Miocene with the oldest Neogene radiolarians representative of the Dorcadospyris alata Zone. Therefore, the initiation of Miocene biosiliceous sedimentation in the northwest Pacific as recorded in sediments along this north-south transect marks the time when each specific site exited from the low-productivity central gyre and moved into the preexisting high-productivity belt at the gyre margins.

Within the period of biosiliceous sedimentation recorded in sediments from the northwest Pacific, a relative measure of radiolarian productivity can be acquired from calculations of the number of radiolarians per gram of sediment. This value was computed in samples from Sites 578, 579, 580, and 581 (Tables 3 through 6). From comparison of these numbers in each of the holes, regional pulses and/or trends in radiolarian productivity can be detected. Specifically, Sites 579 and 580 record a nearly continuous increase in productivity through the early and middle portion of the late Pliocene coincident with the Gauss Chronozone. This trend toward increasing radiolarian productivity levels reaches a maximum early in the Matuyama Chronozone between 2.3 and 2.4 m.y. ago. Radiolarian productivity decreases from these high late Pliocene values, remaining relatively low throughout the early Pleistocene at Sites 578, 579, and 580. Late Pleistocene biosiliceous productivity is somewhat higher than that recorded for the early Pleistocene, with indications of minor productivity increases in the early, middle, and latest Pleistocene.

\section{SYSTEMATICS}

The classification scheme is adapted from Riedel and Sanfilippo (1977) with minor variations following Hays (1970) and Nigrini and Moore (1979). Minimal synonomies of taxa are presented with restric- 
tion to original description and those that reflect current usage. Species arranged alphabetically by family.

Subclass RADIOLARIA Müller 1858

Superorder POLYCYSTINA Ehrenberg 1838, emend. Riedel 1967b

Order SPUMELLARIA Ehrenberg 1875

Family ACTINOMMIDAE Haeckel 1862, emend. Riedel 1967b

Subfamily ACTINOMMIDAE Haeckel 1862, emend. Petrushevskaya and Kozlova, 1972

Genus ACTINOMMA Haeckel 1860, emend. Nigrini 1967

Actinomma medianum Nigrini

(Plate 1, Figs. 4A, 4B)

Actinomma medianum Nigrini, 1967, p. 27, pl. 2, figs. 2a, 2b; 1970 , p. 167, pl. 1, fig. 10; Nigrini and Moore, 1979, p. S31, pl. 3, figs. 5,6 .

\section{Genus DRUPPATRACTUS Haeckel 1887 \\ Druppatractus acquilonius Hays \\ (Plate 4, Figs. 1A, 1B)}

Druppatractus acquilonius Hays, 1970, p. 214, pl. 1, figs. 4, 5. Stylacontarium acquilonium (Hays), Kling, 1973, p. 634, pl. 1, figs. 17-20, pl. 14, figs. 1-4; Ling, 1973, p. 777 , pl. 1, figs. $6,7$. Remarks: Although most recent studies place this species in the genus Stylacontarium, this chapter used the original genus description given by Hays.

\section{Genus SPHAEROPYLE Dreyer 1889 \\ Sphaeropyle langii Dreyer \\ (Plate 5, Figs. 3A, 3B)}

Sphaeropyle langii Dreyer, 1889, p. 13, pl. 4, fig. 54; Kling, 1973, p. 634 , pl. 1, figs. 5-10, pl. 13, figs. 6-8; Foreman, 1975, p. 618, pl. 9 , figs. 30,31 .

\section{Sphaeropyle robusta Kling emend. Foreman}

(Plate 5, Figs. 4A, 4B)

Sphaeropyle robusta Kling, 1973, p. 634, pl. 1, fig. 12, pl. 6, figs. 613; Foreman, 1975, p. 618, pl. 9, figs. 24-26.

\section{Genus STYLATRACTUS Haeckel 1887}

Stylatractus universus Hays

(Plate 4, Figs. 2A, 2B)

Stylatractus sp. Hays, 1965, p. 167, pl. I, fig. 6.

Stylatractus universus Hays, 1970, p. 215, pl. 1, figs 1, 2; Kling, 1971, p. 1086, pl. 1, fig. 6 .

Axoprunum angelinum (Campbell and Clark), Kling, 1973, p. 634, pl. 1, figs. 14-17; Ling, 1973, p. 777, pl. 1, figs. 1-4; Foreman, 1975, p. 618, pl. 9 , figs. 28,29 ; Sakai, 1980 , p. 704 , pl. 2 , figs. 1a, 1 b. Remarks: Although most recent studies place this species in the genus Axoprunum, this chapter used the genus designation of Hays 1970.

Subfamily ARTISCINAE Haeckel 1881, emend. Riedel 1967b Genus DIARTUS Sanfilippo and Riedel 1980

Diartus hughesi (Campbell and Clark)

(Plate 8, Figs. 3A, 3B)

Ommatocampe hughesi Campbell and Clark, 1944, p. 23, pl. 3, fig. 12.

Ommatartus hughesi (Campbell and Clark), Riedel and Sanfilippo, 1970 , p. 521; Kling, 1973, p. 634, pl. 7, fig. 6; Foreman, 1975, p. 619 , pl. 8 , fig. 3; Sakai, 1980, p. 708 , pl. 4, figs. 3a, 3b.

Diartus hughesi (Campbell and Clark), Sanfilippo and Riedel, 1980, p. 1010, pl. 1, fig. i.

\section{Diartus petterssoni (Riedel and Sanfilippo)}

(Plate 8, Figs. 4A, 4B)

Cannartus(?) petterssoni Riedel and Sanfilippo, 1970, p. 520, pl. 14, fig. 3; Kling, 1973, p. 634, pl. 7, fig. 8; Sakai, 1980, p. 705, pl. 4, figs. $4 a, 4 b, 6 a, 6 b, 7 a, 7 b$.
Diartus petterssoni (Riedel and Sanfilippo), Sanfilippo and Riedel, 1980 , p. 1010, pl. 1, fig. h.

\section{Genus DIDYMOCYRTIS Haeckel 1860 \\ Didymocyrtis antepenultima (Riedel and Sanfilippo)}

(Plate 8, Fig. 2)

Ommatartus antepenultimus Riedel and Sanfilippo, 1970, p. 521, pl. 14, fig. 14; Westberg and Riedel, 1978, p. 22, pl. 2, figs. 4, 5; Sakai, 1980 , p. 708 , pl. 3, figs. 3a, 3b.

Didymocyrtis antepenultima (Riedel and Sanfilippo), Sanfilippo and Riedel, 1980, p. 1010.

\section{Didymocyrtis laticonus (Riedel) \\ (Plate 8, Fig. 5)}

Cannartus laticonus Riedel, 1959, p. 291, pl. 1, fig. 5; Westberg and Riedel, 1978, p. 20, pl. 2, figs. 1-3.

Didymocyrtis laticonus (Riedel), Sanfilippo and Riedel, 1980, p. 1010, pl. 1, fig. e.

\section{Didymocyrtis penultima (Riedel)}

(Plate 8, Fig. 1)

Panarium penultimum Riedel, 1957, p. 76, pl. 1, fig. 1

Ommartartus penultimus (Riedel), Westberg and Riedel, 1978, p. 22, pl. 2, figs. 6-8; Sakai, 1980, p. 708, pl. 3, figs. 3a, 3b, 4a, 4b, 5a, 5 b.

Didymocyrtis penultima (Riedel), Sanfilippo and Riedel, 1980, p. 1010, pl. 1, fig. f.

\section{Didymocyrtis tetrathalamus (Haeckel)}

(Plate 1, Figs. 2A, 2B)

Panartus tetrathalamus Haeckel, 1887, p. 378 , pl. 40, fig. 3.

Ommatartus tetrathalamus (Haeckel), Riedel and Sanfilippo, 1971, p. 1588, pl. 1C, figs. 5-7; Sakai, 1980, p. 708, pl. 3, figs. 1a, 1b.

Didymocyrtis tetrathalamus (Haeckel), Sanfilippo and Riedel, 1980, p. 1010, pl. 1, fig. g.

Subfamily SATURNALINAE Deflandre 1953

Genus SATURNALIS Haeckel 1881, emend. Nigrini 1967

Saturnalis circularis Haeckel

(Plate 3, Fig. 1)

Saturnalis circularis Haeckel, 1887, p. 131; Nigrini, 1967, p. 24, pl. 1, fig. 9; Kling, 1973, p. 634, pl. 1, figs. 21-25, pl. 7, figs. 1-5; Ling, 1973, p. 777, pl. 1, fig. 5; Sakai, 1980, p. 709, pl. 6, fig. 14.

\section{Family LITHELIIDAE Haeckel 1862 \\ Genus LARCOSPIRA Haeckel 1887 \\ Larcospira quadrangula Haeckel \\ (Plate 3, Fig. 9)}

Larcospira quadrangula Haeckel, 1887, p. 696, pl. 49, fig. 3; Benson, 1966 , p. 266, pl. 18, figs. 7, 8; Nigrini and Moore, 1979, p. S133, pl. 17 , fig. 2.

Family PHACODISCIDAE Haeckel 1881 Genus HELIODISCUS Haeckel 1862, emend. Nigrini 1967

\section{Heliodiscus asteriscus Haeckel}

(Plate 3, Fig. 8)

Heliodiscus asteriscus Haeckel, 1887, p. 445, pl. 33, fig. 8; Nigrini, 1967 , p. 32. pl. 3, figs. 1a, 1b; Nigrini and Moore, 1979, p. S73, pl. 9 , figs. 1,2 .

Family SPONGODISCIDAE Haeckel 1862, emend. Riedel 1967b Genus EUCHITONIA Ehrenberg 1860, emend. Nigrini 1967

\section{Euchitonia sp.}

(Plate 2, Fig. 3)

Euchitonia elegans (Ehrenberg), Nigrini, 1967, p. 39, pl. 4, figs. 2a, 2b; Nigrini and Moore, 1979, p. S83, pl. 11, figs. 1a, 1b. 
Euchitonia furcata Ehrenberg, 1872a, p. 289, pl. IV, fig. 6; Ling and Anikouchine, 1967 , p. 1484, pl. 189, figs. 1, 2, 5-7, pl. 190, figs. 1, 2, 5-7; Nigrini and Moore, 1979, p. S85, pl. 11, figs. 2a, 2b.

Remarks: Most specimens present were Euchitonia furcata, with only very rare occurrences of Euchitonia elegans.

Genus SPONGASTER Ehrenberg 1860, emend. Riedel and Sanfilippo, 1971

Spongaster tetras irregularis Nigrini (Plate 3, Fig. 5)

Spongaster tetras irregularis Nigrini, 1967 , p. 43, pl. 5, fig. 2; Foreman, 1975, p. 619, pl. 9, fig. 27; Sakai, 1980, p. 709, pl. 6, fig. 2.

\section{Genus SPONGOPYLE Dreyer 1889}

Spongopyle osculosa Dreyer

(Plate 3, Fig. 2)

Spongopyle osculosa Dreyer, 1889, p. 42, pl. 11, figs. 99, 100; Nigrini and Moore, 1979, p. S115, pl. 15, fig. 1.

\section{Genus SPONGOTROCHUS Haeckel 1860 \\ Spongotrochus(?) venustum (Bailey) \\ (Plate 2, Figs. 1A, 1B)}

Perichlamydium venustum Bailey, 1856, p. 5, pl. 1, figs. 16, 17.

Stylochlamydium venustum (Bailey), Haeckel, 1887, p. 515; Ling, 1973, p. 778 , pl. 1 , fig. 8 .

Spongotrochus(?) venustum (Bailey), Nigrini and Moore, 1979, p. S119, pl. 15 , figs. $3 \mathrm{a}, 3 \mathrm{~b}$.

Spongotrochus(?) glacialis Popofsky, Riedel, 1958, p. 227, pl. 2, figs.

1, 2; Kling, 1973, p. 635, pl. 2, figs. 4-6.

Remarks: Included all the above forms in the counts of Spongotrochus(?) venustum.

\section{Family SPONGURIDAE Haeckel 1862, emend. Petrushevskaya 1975 \\ Genus SPONGOCORE Haeckel 1887 \\ Spongocore puella Haeckel \\ (Plate 2, Fig. 5)}

Spongocore puella Haeckel, 1887, p. 347, pl. 48, fig. 6; Benson, 1964, pl. 1, fig. 21; Kling, 1973, p. 635, pl. 7, figs. 18-22; Nigrini and Moore, 1979, p. S69, pl. 8, figs. 5a-5c.

\section{Genus SPONGURUS Haeckel 1860 \\ Spongurus pylomaticus Riedel \\ (Plate 2, Fig. 4)}

Spongurus pylomaticus Riedel, 1958, p. 226, pl. 1, figs. 10, 11; Petrushevskaya, 1967, p. 32, figs. 16I, 16II; Nigrini and Moore, 1979 , p. S65, pl. 8, figs. 3a, 3b.

Spongurus(?) pylomaticus Riedel, Petrushevskaya, 1975, p. 577, pl. 7, fig. 4 , pl. 37 , fig. 7 .

ORDER NASSELLARIA Ehrenberg 1875 Suborder CYRTIDA Haeckel 1862, emend. Petrushevskaya 1971 Family ARTOSTROBIIDAE Riedel 1967a, emend. Foreman 1973b Genus BOTRYOSTROBUS Haeckel 1887, emend. Nigrini 1977

Botryostrobus aquilonaris (Bailey) (Plate 1, Fig. 1)

Eucyrtidium aquilonaris Bailey, 1856, p. 4, pl. 1, fig. 9.

Eucyrtidium tumidulum Bailey(?), Kling, 1971, pl. 1, fig. 2.

Botryostrobus aquilonaris (Bailey), Nigrini, 1977, p. 246, pl. 1, fig. 1; Nigrini and Moore, 1979, p. N99, pl. 27, fig. 1.

\section{Genus PHORMOSTICHOARTUS Campbell 1951, emend.} Nigrini 1977

\section{Phormostichoartus corbula (Harting)}

(Plate 3, Fig. 6)

Lithocampe corbula Harting, 1863, p. 12, pl. 1, fig. 21.

Siphocampe corbula (Harting), Nigrini, 1967, p. 85, pl. 8, fig. 5, pl. 9, fig. 3.
Phormostichoartus corbula (Harting), Nigrini, 1977, p. 252, pl. 1. fig. 10; Nigrini and Moore, 1979, p. N103, pl. 27, fig. 3.

Family CARPOCANIIDAE Haeckel 1881, emend. Riedel 1967b Genus CARPOCANISTRUM Haeckel 1887, emend. Riedel and Sanfilippo, 1971

\section{Carpocanistrum spp.}

(Plate 3, Fig. 7)

Carpocanium petalospyris Benson, 1966, p. 434, pl. 29, figs. 9, 10, 25.

Carpocanium spp. Nigrini, 1970, p. 171, pl. 4, figs. 4-6.

Carpocanistrum spp. Riedel and Sanfilippo, 1971, p. 1596, pl. 1G, figs. $1-6,8-13$, pl. 2F, figs. 5, 6, pl. 3D, figs. 1, 2, 6, 7, 9; Nigrini and Moore, 1979, p. N23, pl. 21, figs. 1a-1c.

Family PTEROCORYIDAE Haeckel 1881, emend. Riedel 1967b Genus LAMPROCYCLAS Haeckel 1881, emend. Petrushevskaya and Kozlova, 1972

Lamprocyclas maritalis (Plate 3, Fig. 3)

Lamprocyclas maritalis Haeckel, 1887, p. 1390, pl. 74, figs. 13, 14. Lamprocyclas maritalis maritalis Haeckel, Nigrini, 1967, p. 74, pl. 7, fig. 5; Nigrini and Moore, 1979, p. N75, pl. 25, fig. 4.

Lamprocyclas maritalis polypora Nigrini, 1967. p. 76, pl. 7, fig. 6; $\mathrm{Ni}-$ grini and Moore, 1979, p. N77, pl. 25, fig. 5.

Remarks: Counts of these two subspecies of Lamprocyclas maritalis (maritalis and polypora) were combined in this study.

\section{Genus LAMPROCYRTIS Kling 1973 \\ Lamprocyrtis heteroporos (Hays) \\ (Plate 6, Figs. 3A, 3B)}

Lamprocyclas heteroporos Hays, 1965, p. 179, pl. 3, fig. 1.

Lamprocyrtis heteroporos (Hays), Kling, 1973, p. 639, pl. 5, figs. 19, 20; Foreman, 1975, p. 620, pl. 9, fig. 21; Sakai, 1980, p. 711, pl. 9 , figs. 9, 10, 11a, 11b.

\section{Lamprocyrtis neoheteroporos Kling}

(Plate 6, Fig. 2)

Lamprocyrtis neoheteroporos $\mathrm{Kling}$, 1973, p. 639, pl. 5, figs. 17, 18, pl. 15, figs. 4, 5; Foreman, 1975, p. 620, pl. 9, fig. 12; Sakai, 1980, p. 711 , pl. 9, figs. $9 \mathrm{a}, 9 \mathrm{~b}$.

\section{Lamprocyrtis nigriniae (Caulet)}

(Plate 6, Fig. 1)

Conarchnium nigriniae Caulet, 1971, p. 3, pl. 3, figs. 1-4, pl. 4, figs. $1-4$.

Lamprocyrtis haysi Kling, 1973, p. 639, pl. 5, figs. 15, 16, pl. 15, figs. 1-3; Foreman, 1975, p. 620, pl. 9, fig. 22; Sakai, 1980, p. 711, pl. 7 , figs. $8 \mathrm{a}, 8 \mathrm{~b}$.

Lamprocyrtis nigriniae (Caulet), Nigrini and Moore, 1979, p. N81, pl. 25 , fig. 7 .

\section{Genus PTEROCORYS Haeckel 1881 \\ Pterocorys zancleus (Müller) \\ (Plate 3, Fig. 4)}

Eucyrtidium zanclaeum Müller, 1858, p. 41, pl. 6, figs. 1-3.

Theoconus zancleus (Müller), Benson, 1966, p. 482, pl. 33, fig. 4; Kling, 1973 , p. 639 , pl. 5, figs. 9-11.

Pterocorys zancleus (Müller), Nigrini and Moore, 1979, p. N89, pl. 25 , figs. $11 \mathrm{a}, 11 \mathrm{~b}$

\section{Genus THEOCORYTHIUM Haeckel \\ Theocorythium trachelium dianae (Haeckel)} (Plate 5, Fig. 1)

Theocorys dianae Haeckel, 1887 , p. 1416, pl. 69, fig. 11.

Theocorythium trachelium dianae (Haeckel), Nigrini, 1967, p. 77, pl. 8, figs. 1a, 1b, pl. 9, figs. 1a, 1b; Foreman, 1975, p. 620, pl. 9, fig. 13; Sakai, 1980, p. 711 , pl. 10, figs. 1a, 1 b. 
Theocorythium trachelium trachelium (Ehrenberg)

$$
\text { (Plate 5, Fig. 2) }
$$

Eucyrtidium trachelius Ehrenberg, 1872b, p. 312.

Theorcorythium trachelium trachelium (Ehrenberg), Nigrini, 1967, p. 79 , pl. 8, fig. 2, pl. 9, fig. 2; Foreman, 1975, p. 620, pl. 9, fig. 12; Sakai, 1980 , p. 711 , pl. 10 , figs. $2 a, 2 b$.

\section{Family THEOPERIDAE Haeckel 1881, emend. Riedel 1967b Genus CLATHROCYCLAS Haeckel 1881}

Clathrocyclas spp.

(Plate 1, Figs. 5A, 5B)

Remarks: The description by Kling (1973) for this group was followed in this study.

\section{Genus CORNUTELLA Ehrenberg 1838 \\ Cornutella profunda Ehrenberg \\ (Plate 1, Fig. 3)}

Cornutella clathrata $\beta$ profunda Ehrenberg, 1856, pl. 35B, fig. 21; Bailey, 1856, p. 2, pl. 1, fig. 23.

Cornutella profunda Ehrenberg, 1858, p. 31; Riedel, 1958, p. 232, pl. 3, figs. 1, 2; Nigrini, 1967, p. 60, pl. 6, figs. 5a-5c; Kling, 1973, p. 635 , pl. 3 , figs. $1-4$, pl. 9 , figs. $8-17$.

\section{Genus CYCLADOPHORA Ehrenberg 1847 \\ Cycladophora davisiana Ehrenberg \\ (Plate 1, Figs. 6A, 6B)}

Cycladophora(?) davisiana Ehrenberg, 1861, p. 297.

Theocalyptra davisiana (Ehrenberg), Riedel, 1958, p. 239, pl. 4, figs. 2, 3; Kling, 1973, p. 638, pl. 3, figs. 9-12; Nigrini and Moore, 1979 , p. N59, pl. 24, figs. 2a, 2 b.

Cycladophora davisiana Ehrenberg, Petrushevskaya, 1967, p. 122, pl. 69 , figs. I-VII; Ling, 1973, p. 780 , pl. 2, fig. 2; Sakai, 1980, p. 709 , pl. 6 , figs. $6,7 a, 7 b, 8 a, 8 b$.

Cycladophora davisiana var. cornutoides Petrushevskaya (Plate 2, Figs. 2A, 2B)

Cycladophora davisiana var. cornutoides Petrushevskaya, 1967, p. 124, pl. 71, figs. I-III; Ling, 1973, p. 780, pl. 2, fig. 3.

\section{Genus CYRTOCAPSELLA Haeckel 1887, emend. Sanfilippo and Riedel 1970 \\ Cyrtocapsella cornuta Haeckel}

(Plate 8, Fig. 8)

Cyrtocapsa (Cyrtocapsella) cornuta Haeckel, 1887, p. 1513, pl. 78, fig. 9.

Cyrtocapsella cornuta Haeckel, Sanfilippo and Riedel, 1970, p. 453, pl. 1, figs. 19, 20; Kling, 1973, p. 636, pl. 11, figs. 16-18; Foreman, 1975 , p. 620 , pl. 8 . fig. 8 ; Sakai, 1980 , p. 709 , pl. 8 , figs. 8 a, 8 b.

\section{Cyrtocapsella japonica (Nakaseko)}

(Plate 8, Fig. 7)

Eusyringium japonicum Nakaseko, 1963, p. 193, pl. 4, figs. 1-3. Cyrtocapsella japonica (Nakaseko), Sanfilippo and Riedel, 1970, p. 452 , pl. 1, figs. $13-15$; Kling, 1973 , p. 636 , pl. 11, figs. 19,20 ; Foreman, 1975, p. 620, pl. 8, fig. 7; Sakai, 1980, p. 709, pl. 8, figs. 7a, $7 \mathrm{~b}$.

\section{Cyrtocapsella tetrapera Haeckel}

(Plate 8, Fig. 6)

Cyrtocapsa (Cyrtocapsella) tetrapera Haeckel, 1887, p. 1512, pl. 78, fig. 5 .

Cyrtocapsella tetrapera Haeckel, Sanfilippo and Riedel, 1970, p. 453 , pl. 1, figs. 16-18; Kling, 1973, p. 636, pl. 11, figs. 12-15; Ling, 1973 , p. 780 , pl. 2 , fig. 4 ; Sakai, 1980 , p. 709 , pl. 8 , figs. 5,6 .
Genus EUCYRTIDIUM Ehrenberg 1847, emend. Nigrini 1967

Eucyrtidium acuminatum (Ehrenberg)

(Plate 5, Fig. 5)

Lithocampe acuminatum Ehrenberg, 1844, p. 84.

Eucyrtidium acuminatum (Ehrenberg), Nigrini, 1967, p. 81, pl. 8, figs. 3a, 3b; Kling, 1973, p. 636, pl. 4, figs. 20-23; Nigrini and Moore, 1979, p. N61, pl. 24, figs. 3a, 3b.

\section{Eucyrtidium calvertense Martin}

(Plate 5, Fig. 6)

Eucyrtidium calvertense Martin, 1904, p. 450, pl. 130, fig. 5; Kling, 1973 , p. 636 , pl. 4 , figs. $16,18,19$, pl. 11, figs. 1-5; Foreman, 1975 , p. 620 , pl. 9, fig. 14.

\section{Eucyrtidium matuyamai Hays}

(Plate 5, Figs. 7A, 7B)

Eucyrtidium matuyamai Hays, 1970, p. 213, pl. 1, figs. 7-9; Kling, 1971, p. 1088, pl. 1, fig. 4; Kling, 1973, p. 636, pl. 4, fig. 17; Ling, 1973 , p. 780 , pl. 2, figs. 5, 6; Foreman, 1975, p. 620, pl. 9 , fig. 15 ; Sakai, 1980 , p. 710 , pl. 7 , figs. 1a, 1 b.

Genus LYCHNOCANOMA Haeckel 1887, emend. Foreman 1973b

\section{Lychnocanoma grande (Campbell and Clark)}

(Plate 6, Figs. 4A, 4B)

Lychnocanoma grande Campbell and Clark, 1944, p. 42, pl. 6, figs. 3-6; Kling, 1973, p. 637, pl. 10, figs. 10-14.

\section{Genus PTEROCANIUM Ehrenberg 1847 \\ Pterocanium prismatium Riedel \\ (Plate 7, Figs. 3A, 3B)}

Pterocanium prismatium Riedel, 1957, p. 87, pl. 3, figs. 4, 5; Sakai, 1980, p. 711 , pl. 8 , figs. 15 a, 15 b.

\section{Genus STICHOCORYS Haeckel 1881 \\ Stichocorys delmontensis (Campbell and Clark) \\ (Plate 7, Fig. 2)}

Eucyrtidium delmontense Campbell and Clark, 1944, p. 56, pl. 7, figs. 19, 20.

Stichocorys delmontensis (Campbell and Clark), Sanfilippo and Riedel, 1970 , p. 451 , pl. 1, fig. 9; Kling, 1973, p. 638 , pl. 11, figs. 810; Ling, 1973, p. 781, pl. 2, fig. 12; Foreman, 1975, p. 622, pl. 9 , figs. 5-7; Sakai, 1980, p. 711, pl. 8. fig. 3.

\section{Stichocorys peregrina Riedel)}

(Plate 7, Figs. 1A, 1B)

Eucyrtidium elongatum peregrinum Riedel, 1953, p. 812, pl. 85, fig. 2.

Stichocorys peregrina (Riedel), Sanfilippo and Riedel, 1970, p. 451, pl. 1, fig. 10; Kling, 1973, p. 638, pl. 4, fig. 27, pl. 11, fig. 29, pl. 13, figs. 9, 10; Foreman, 1975, p. 622, pl. 9, figs. 1-4; Sakai, 1980, p. 711 , pl. 8 , figs. 1,2 .

\section{ACKNOWLEDGMENTS}

I thank W. R. Riedel and C. Nigrini for their helpful suggestions for improving an earlier version of this manuscript. A. Wright also reviewed this document, insuring that remarks were in agreement with those stated elsewhere in this volume.

\section{REFERENCES}

Bailey, J. W., 1856. Notice of microscopic forms found in the soundings of the Sea of Kamtschatka-with a plate. Am. J. Sci. Arts, Ser. 2, 22:1-6.

Benson, R. N., 1964. Preliminary report on Radiolaria in Recent sediments of the Gulf of California. In van Andel, T. H., and Shor, G. G., Jr. (Eds.), Marine Geology of the Gulf of California-A Symposium. Mem. Am. Assoc. Pet. Geol., 3:398-400. 
1966. Recent radiolaria from the Gulf of California [Ph.D. dissert.]. University of Minnesota.

Campbell, A. S., 1951. New genera and subgenera of radiolaria. J. Paleontol., 25:527-530.

Campbell, A. S., and Clark, B. L., 1944. Miocene radiolarian faunas from southern California. Spec. Pap. Geol. Soc. Am., 51:1-76.

Caulet, J., 1971. Contribution à l'étude de quelques Radiolaires Nassellaires des bous de la Méditerranée et du Pacifique. Arch. Orig. Centre de Documentation C.N.R.S., Cahiers de Micropaleontologie, Ser. 2(498), 10:1-10.

Deflandre, G., 1953. Radiolaires fossiles. In Grassé, P.-P. (Ed.), Traite de Zoologie (Vol. 1): Paris (Masson), 389-436.

Dreyer, F., 1889. Die Pylombildungen in vergleichend-anatomischer und entwicklungsgeschichtlicher Beziehung bei Radiolarien und bei Protisten überhaupt, nebst System und Beschreibung neuer und der bis jetzt bekannten pylomatischen Spumellarien. Jena. Z. Naturwiss., new Ser. 16, 23:1-138.

Ehrenberg, C. G., 1838. Über die Bildung der Kriedefelsen und des Kriedemergels durch unsichtbare Organismen. Abh. Kgl. Preuss. Akad. Wiss. Berlin Jahrg., pp. 59-147.

1844. Über 2 neue Lager von Gebirgsmassen aus Infusorien als Meeres-Absatz in Nord-Amerika unde eine Vergleichung derselben mit den organischen Kreide-Gebilden in Europa und Afrika. Monatsber. Kgl. Preuss. Akad. Wiss. Berlin Jahrg., pp. 57-97.

1847. Über die mikroskopischen kieselschaligen Polycystinen als mächtige Gebirgsmasse von Barbados ünd uber das Verhältnis der aus mehr als 300 neuen Arten bestehenden ganz eigenthümlichen Formengruppe jener Felsmasse zu den lebenden Thieren und zur Kreidebildung. Eine neue Anregung zur Erforschung der Erdlebens. Monatsber. Kgl. Preuss. Akad. Wiss. Berlin Jahrg., pp. 40-60.

, 1856. Über 2 neue südamerikanische Gebirgsmassen aus mikroskopischen Organismen, eine aus Meeresorganismen in Chile und eine als mit gefritteten Süfswasserorganismen gemischten vulkanischen efsbaren Tuff aus Honduras in Centro-Amerika. Monatsber. Kgl. Preuss. Akad. Wiss, Berlin Jahrg., pp. 425-431. 1858. Kurze Characteristik der 9 neuen Genera und der 105 neuen Species des ägäischen Meeres und des Tiefgrundes des Miffel-Meeres. Monatsber. Kgl. Preuss. Akad. Wiss. Berlin Jahrg., pp. $10-40$.

, 1860. Über den Tiefgrund des stillen Oceans zwischen Californien und den Sandwich-Inseln aus bis 15600' Tiefe nach Lieut. Brooke. Monatsber. Kgl. Preuss. Akad. Wiss. Berlin Jahrg., pp. 819-833.

, 1861. Über die Tiefgrund-Verhältnisse des Oceans am Eingange der Davisstrasse und bei Island. Nebst einem ÜbersichtsKärtchen. Monatsber. Kgl. Preuss. Akad. Wiss. Berlin Jahrg., pp. 275-315.

1872a. Mikrogeologische Studien über das kleinste Leben der Meeres-Tiefgründe aller Zonen und dessen geologischen Einfluss. Abh. Kgl. Preuss. Akad. Wiss. Berlin Jahrg., pp. 131-399. $1872 \mathrm{~b}$. Mikrogeologischen Studien als Zusammenfassung seiner Beobachtungen des kleinsten Lebens der Meeres-Tiefgrunde aller Zonen und dessen geologischen Einfluss. Monatsber. Kgl. Preuss. Akad. Wiss. Berlin Jahrg., pp. 265-322.

, 1875. Fortsetzung der mikrogeologischen Studien als Gesammt-Uebersicht der mikroskopischen Paläontologie gleichartig analysirter Gebirgsarten der Erde, mit specieller Rücksicht auf den Polycystinen-Mergel von Barbados. Abh. Kgl. Preuss. Akad. Wiss. Berlin Jahrg., pp. 1-226.

Foreman, H. P., 1973a. Radiolaria from DSDP Leg 20. In Heezen, B. C., MacGregor, I. D., et al., Init. Repts. DSDP, 20: Washington (U.S. Govt. Printing Office), 249-306.

1973b. Radiolaria of Leg 10 with systematics and ranges for the families Amphipyndacidae, Artostrobiidae, and Theoperidae. In Worzel, J. L., Bryant, W., et al., Init. Repts. DSDP, 10: Washington (U.S. Govt. Printing Office), 407-474. 1975. Radiolaria from the North Pacific, Deep Sea Drilling Project, Leg 32. In Larson, R. L., Moberly, R., et al., Init. Repts. DSDP, 32: Washington (U.S. Govt. Printing Office), 579-676.

Haeckel, E., 1860. Über neue, lebende Radiolarien des Mittelmeeres und legte die dazu gehörigen Abbildungen. Monatsber. Kgl. Preuss. Akad. Wiss. Berlin Jahrg., pp. 794-817. (Reimer)
1881. Entwurf eines Radiolarien-Systems auf Grund von Studien der Challenger-Radiolarien. Jena. Z. Naturwiss, 15: 418-472.

1887. Report on the Radiolaria collected by H.M.S. Challenger during the years 1873-76. Report on the Scientific Results of the Voyage of the H.M.S. Challenger. Zoology, 18:1-1803.

Harting, P., 1863. Bijdrage tot de kennis der mikroskopische faune en flora van de Banda-Zee, naar aanleiding van een onderzoek van eenige door diepzeeloodingen van 990 tot 4000 vademen uit die zee opgebragte gronden. Verh. K. Akad. Wet. Amsterdam Afd. Natuurkd., 10:1-34.

Hays, J. D., 1965. Radiolaria and late Tertiary and Quaternary history of Antarctic seas. In Llano, G. A. (Ed.), Biology of the Antarctic Seas II (Vol. 5): Washington (Am. Geophys. Union), 125-184.

1970. Stratigraphy and evolutionary trends of radiolaria in North Pacific deep-sea sediments. In Hays, J. D. (Ed.), Geological Investigations of the North Pacific. Mem. Geol. Soc. Am., 126: $185-218$.

Hays, J. D., and Shackleton, N. J., 1976. Globally synchronous extinction of the radiolarian Stylatractus universus. Geology, 4: 649-652.

Kellogg, D. E., and Hays, J. D., 1975. Microevolutionary patterns in late Cenozoic radiolaria. Paleontology, 1:150-160.

Kling, S. A., 1971. Radiolaria: Leg 6 of the Deep Sea Drilling Project. In Fischer, A. G., Heezen, B. C., et al., Init. Repts. DSDP, 6: Washington (U.S. Govt. Printing Office), 1069-1117.

1973. Radiolaria from the eastern North Pacific, Deep Sea Drilling Project, Leg 18. In Kulm, L. D., von Huene, R., et al., Init. Repts. DSDP, 18: Washington (U.S. Govt. Printing Office), 617-672.

Ling, H.-Y., 1973. Radiolaria: Leg 19 of the Deep Sea Drilling Project. In Creager, J. S., Scholl, D. W., et al., Init. Repts. DSDP, 19: Washington (U.S. Govt. Printing Office), 777-798.

1980. Radiolarians from the Emperor Seamounts of the Northwest Pacific: Leg 55 of the Deep Sea Drilling Project. In Jackson, E. D., Koizumi, I., et al., Init. Repts. DSDP, 55: Washington (U.S. Govt. Printing Office), 365-374.

Ling, H.-Y., and Anikouchine, W. A., 1967. Some Spumellarian radiolaria from the Java, Philippine and Mariana trenches. J. Paleontol., 41:1481-1491.

Martin, G. C., 1904. Radiolaria: Baltimore (Maryland Geol. Survey), pp. 447-459.

Moore, T. C., Jr., 1973. Method of randomly distributing grains for microscopic examination. J. Sediment. Petrol., 43:904-906.

Morley, J. J., and Shackleton, N. J., 1978. Extension of the radiolarian Stylatractus universus as a biostratigraphic datum to the Atlantic Ocean. Geology, 6:309-311.

Müller, J., 1858 Über die Thalassicollen, Polycystinen und Acanthometren des Mittelmeeres. Abh. Kgl. Preuss. Akad. Wiss. Berlin Jahrg., pp. 1-62.

Nakaseko, K., 1963. Neogene Cyrtoidea (Radiolaria) from the Isozaki Formation in Ibaraki Prefecture, Japan. Osaka Univ., Coll. General Education, Sci. Rept., 12:165-198.

Nigrini, C., 1967. Radiolaria in pelagic sediments from the Indian and Atlantic Ocean. Bull. Scripps. Inst. Oceanogr., 11:1-125. 1970. Radiolarian assemblages in the North Pacific and their application to a study of Quaternary sediments in Core V20-130. In Hays, J. D. (Ed.), Geological Investigations of the North Pacific. Mem. Geol. Soc. Am., 126:139-184.

1977. Tropical Cenozoic Artostrobiidae (Radiolaria). Micropaleontology, 23:241-269.

Nigrini, C., and Moore, T. C., Jr., 1979. A guide to modern Radiolaria. Spec. Publ. Cushman Found. Foraminiferal Res. 16.

Petrushevskaya, M. G., 1967. Radiolyarii otryador Spumellaria i Nassellaria antarkticheskoi oblasti (Antarctic spumelline and nasselline radiolarians). In Andriyashev, A. P., and Ushakov, P. V. (Eds.), Rez. Biol. Issed. Sov. Antarkt. Eksped. 1955-58, 3:5-187. 1971. On the natural system of polycystine Radiolaria (Class Sarcodina). In Farinacci, A. (Ed.), Proc. II Plankt. Conf.: Rome (Tecnoscienza), pp. 981-992.

1975. Cenozoic radiolarians of the Antarctic, Leg 29, DSDP. In Kennett, J. P., Houtz, R. E., et al., Init. Repts. DSDP, 29: Washington (U.S. Govt. Printing Office), 541-676.

Petrushevskaya, M. G., and Kozlova, G. E., 1972. Radiolaria: Leg 14, Deep Sea Drilling Project. In Hayes, D. E., Pimm, A. C., et al., 
Init. Repts. DSDP, 14: Washington (U.S. Govt. Printing Office), 495-648.

Reynolds, R. A., 1980. Radiolarians from the western North Pacific, Leg 57, Deep Sea Drilling Project. In Scientific Party, Init. Repts. DSDP, 56, 57, Pt. 2: Washington (U.S. Govt. Printing Office), 735-770.

Riedel, W. R., 1953. Mesozoic and late Tertiary Radiolaria of Rotti. J. Paleontol., 27:805-813.

, 1957. Radiolaria: a preliminary stratigraphy. Rep. Swed. Deep Sea Exped. 1947-1948, 6:59-96.

, 1958. Radiolaria in Antarctic sediments. Rep. B.A.N.Z. Antarctic Res. Exped., Ser. B., 6:217-255.

1959. Oligocene and Lower Miocene Radiolaria in tropical Pacific sediments. Micropaleontology, 5:285-302.

1967a. Some new families of Radiolaria. Proc. Geol. Soc. London, 1640:148-149.

1967b. Protozoa [Subclass Radiolaria]. In Harland, W. B., Holland, C. H., House, M. R., Hughes, N. F., Reynolds, A. B., et al. (Eds.), The Fossil Record: London (Geol. Soc. London), pp. 291-298.

Riedel, W. R., and Sanfilippo, A., 1970. Radiolaria, Leg 4, Deep Sea Drilling Project. In Bader, R. G., Gerard, R. D., et al., Init. Repts. DSDP, 4: Washington (U.S. Govt. Printing Office), 503-575.

1971. Cenozoic Radiolaria from the western tropical Pacific, Leg 7. In Winterer, E. L., Riedel, W. R., et al., Init. Repts.
DSDP, 7, Pt. 2: Washington (U.S. Govt. Printing Office), 1529-1672.

, 1977. Cenozoic Radiolaria. In Ramsey, A. T. S. (Ed.), Oceanic Micropaleontology (Vol. 2): London (Academic Press), 847-912.

, 1978. Stratigraphy and evolution of tropical Cenozoic radiolarians. Micropaleontology, 24:61-96.

Sakai, T., 1980. Radiolarians from Sites 434, 435, and 436, Northwest Pacific, Leg 56, Deep Sea Drilling Project. In Scientific Party, Init. Repts. DSDP, 56, 57, Pt. 2: Washington (U.S. Govt. Printing Office), 695-734.

Sanfilippo, A., and Riedel, W. R., 1970. Post-Eocene "closed" theoperid radiolarians. Micropaleontology, 16:446-462.

, 1980. A revised generic and suprageneric classification of the Articins (Radiolaria). J. Paleontol., 54: 1008-1012.

Schaaf, A., 1981. Late Early Cretaceous Radiolaria from Deep Sea Drilling Project Leg 62. In Thiede, J., Vallier, T. L., et al., Init. Repts. DSDP, 62: Washington (U.S. Govt. Printing Office), 419-470.

Westberg, M. J., and Riedel, W. R., 1978. Accuracy of radiolarian correlations in the Pacific Miocene. Micropaleontology, 24:1-23.

Date of Initial Receipt: 28 September 1983

Date of Acceptance: 9 April 1984 


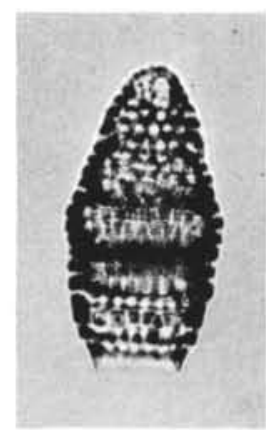

1

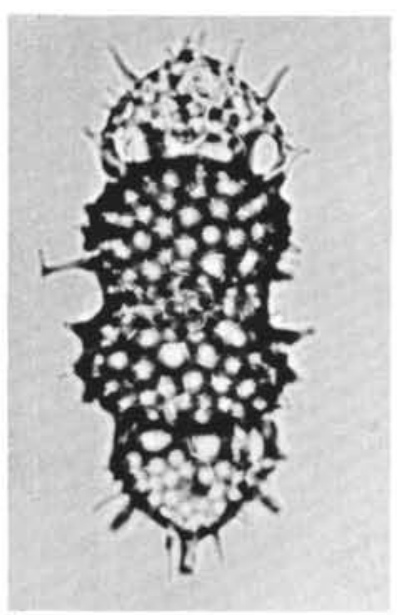

$2 A$

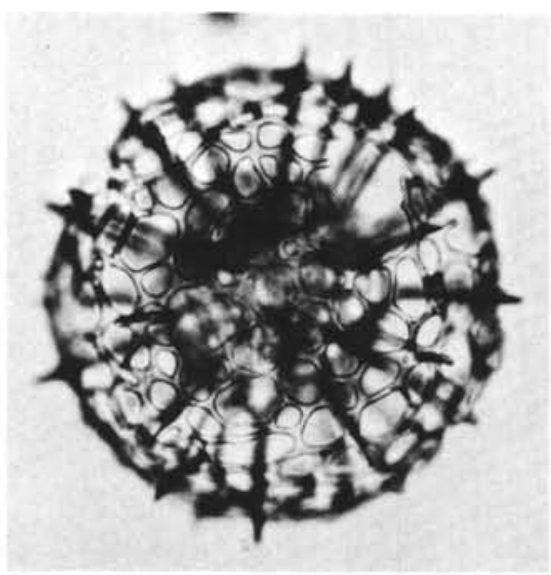

$4 \mathrm{~A}$

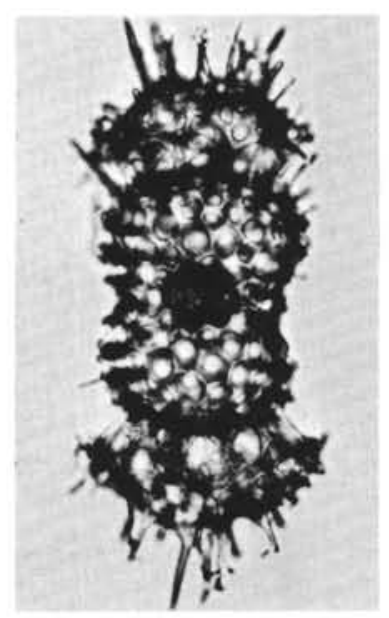

2B

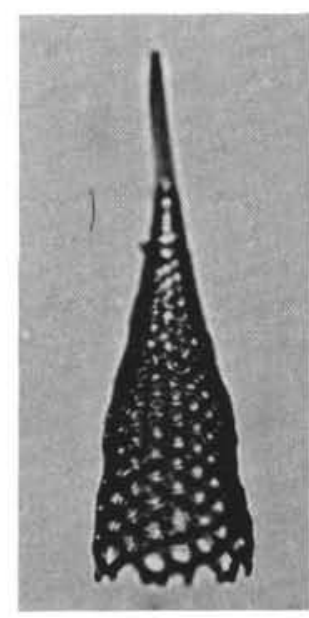

3

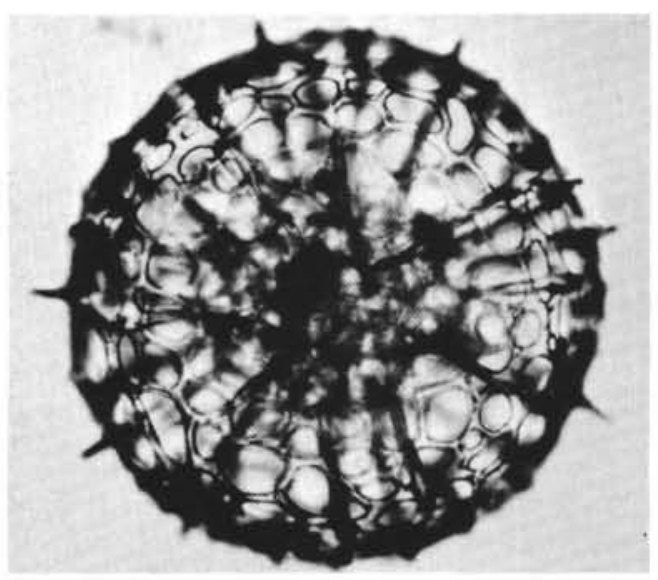

4B

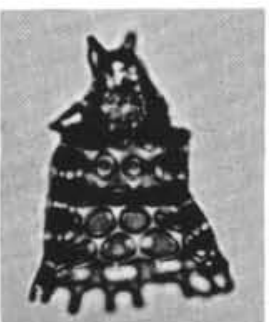

$6 A$

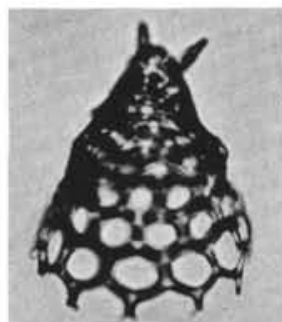

$6 \mathrm{~B}$

Plate 1. 1. Botryostrobus aquilonaris (Bailey), Sample 577-1-1, 5-6 cm. 2A. Didymocyrtis tetrathalamus (Haeckel), Sample 577-1-1, 5-6 cm. 2B. Didymocyrtis tetrathalamus (Haeckel), Sample 578-7-1, 100-102 cm. 3. Cornutella profunda Ehrenberg, Sample 580-11,CC (20-22 cm). 4A. Actinomma medianum Nigrini, Sample 578-1-1, 10-11 cm. 4B. Actinomma medianum Nigrini, Sample 578-7-1, 100-102 cm. 5A. Clathrocyclas spp., Sample 577-1-1, 5-6 cm. 5B. Clathrocyclas spp., Sample 578-7-1, 100-102 cm. 6A. Cycladophora davisiana Ehrenberg, Sample 579A-1,CC (17-19 cm). 6B. Cycladophora davisiana Ehrenberg, Sample 578-7-1, 100-102 cm. (Scale bar equals $100 \mu \mathrm{m}$; magnifications are $\times 200$.) 


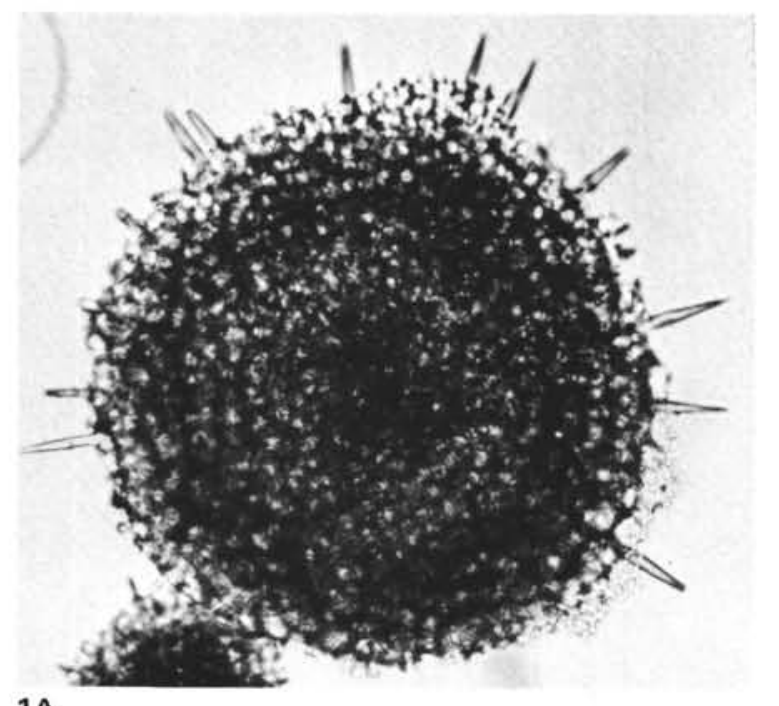

$1 \mathrm{~A}$

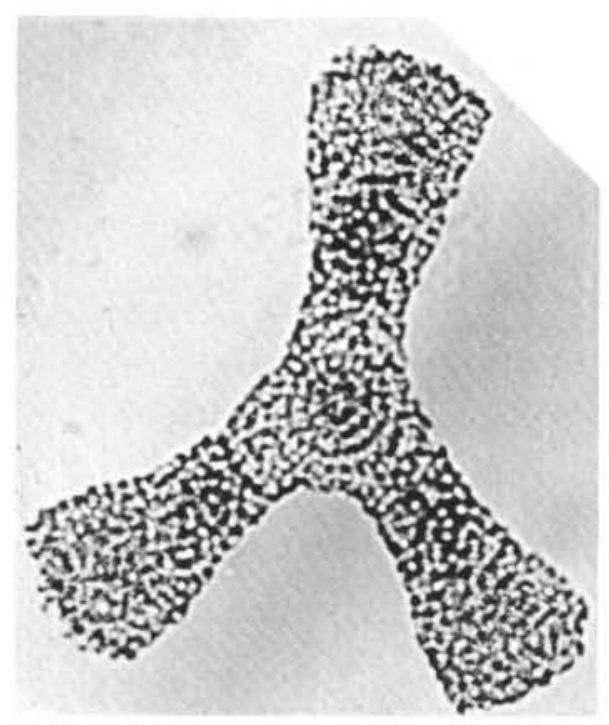

3

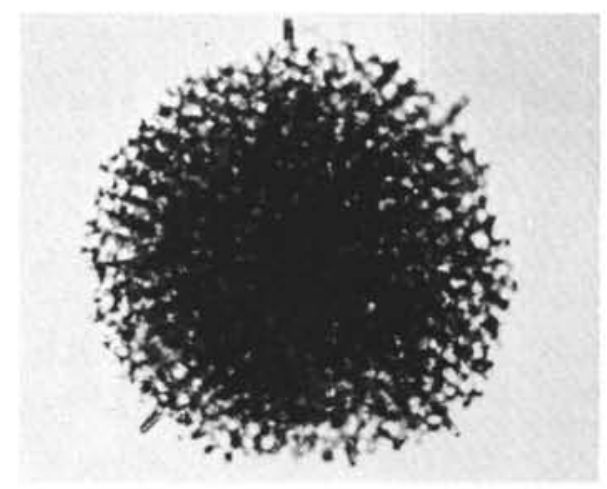

1B

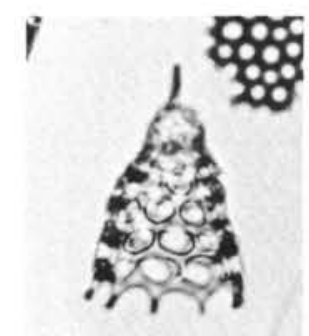

$2 A$

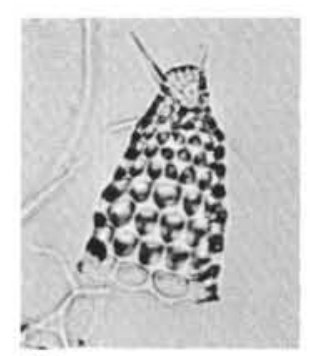

2B

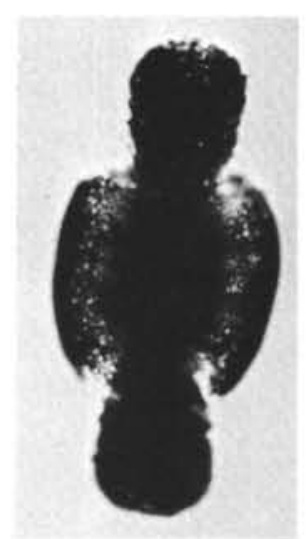

5

Plate 2. 1A. Spongotrochus(?) venustum (Bailey), Sample 580-10,CC (35-37 cm). 1B. Spongotrochus(?) venustum Bailey, Sample 578-7-1, 100$102 \mathrm{~cm}$. 2A. Cycladophora davisiana var. cornutoides Petrushevskaya, Sample 581-3-1, 70-72 cm. 2B. Cycladophora davisiana var. cornutoides Petrushevskaya, Sample 577-1-1, 5-6 cm. 3. Euchitonia sp., Sample 579A-1,CC (17-19 cm). 4. Spongurus pylomaticus Riedel, Sample 579-2,CC (11-13 cm). 5. Spongocore puella Haeckel, Sample 578-12-1, 15-17 cm. (Scale bar equals $100 \mu$ m; magnifications are $\times 200$.) 


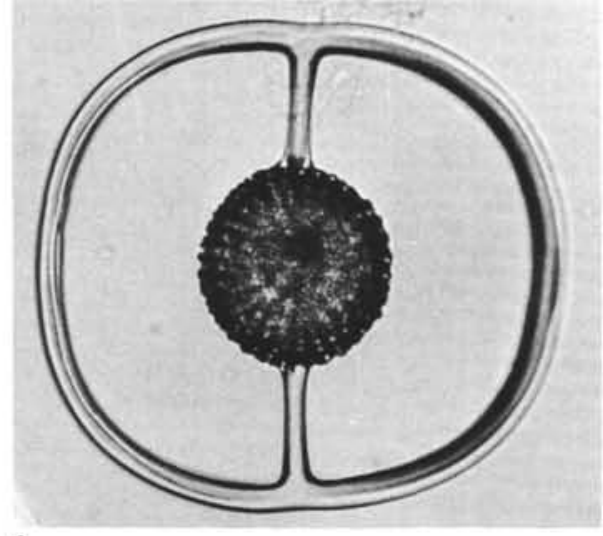

1

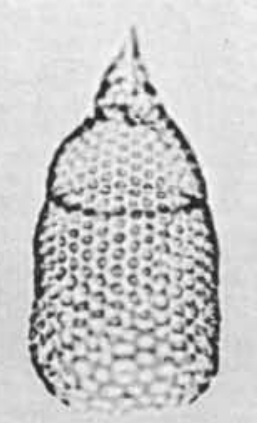

\section{5}
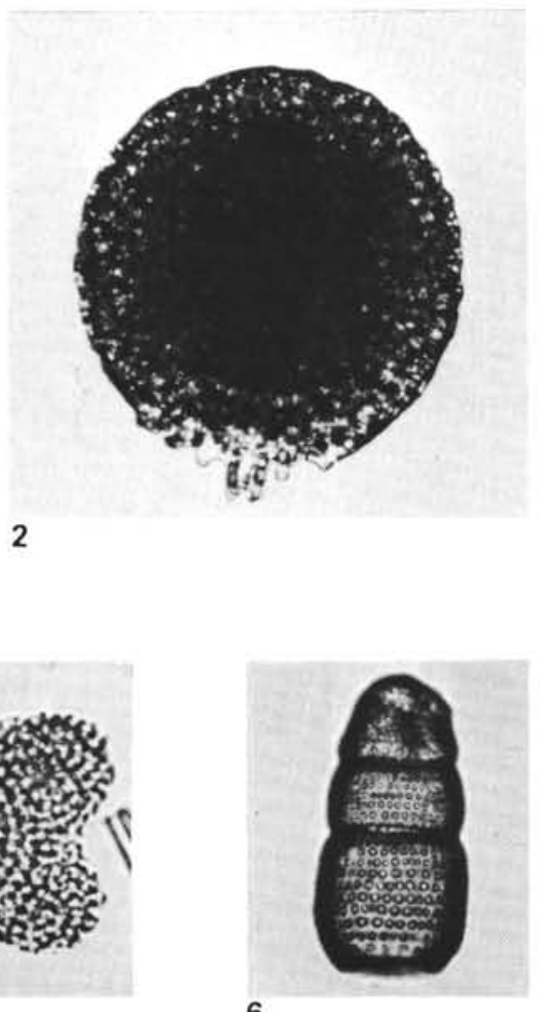

6

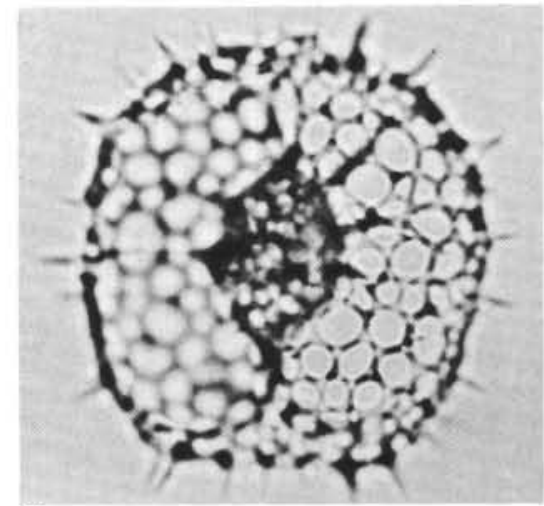

9

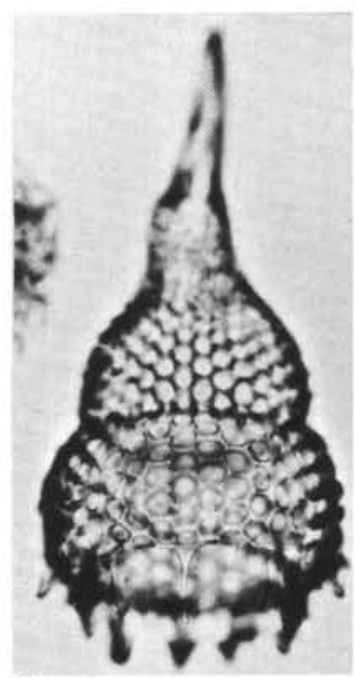

3

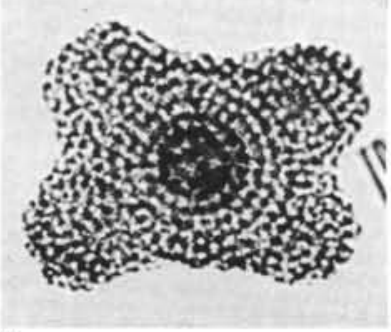

4

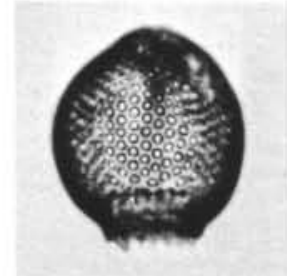

7

8

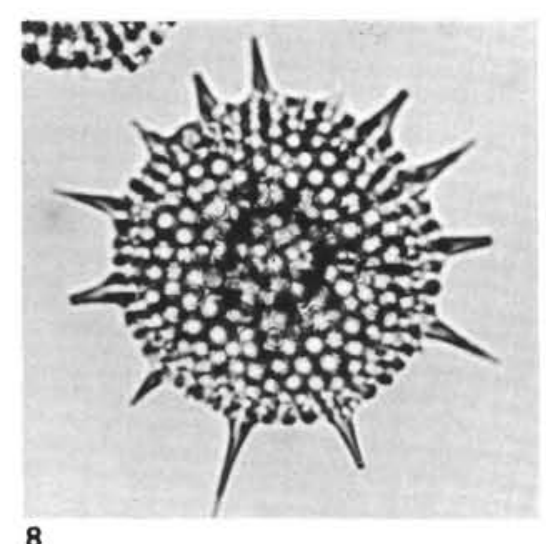

Plate 3. 1. Saturnalis circularis Haeckel, Sample 579-2,CC (11-13 cm). 2. Spongopyle osculosa Dreyer, Sample 578-7-1, 100-102 cm. 3. Lamprocyclas maritalis, Sample $578-7-1,100-102 \mathrm{~cm}$. 4. Pterocorys zancleus (Müller), Sample 577-1-1, 5-6 cm. 5. Spongaster tetras irregularis Nigrini, Sample 578-1-1, 10-11 cm. 6. Phormostichoartus corbula (Harting), Sample 581-8-1, 70-72 cm. 7. Carpocanistrum spp., Sample $578-7-1,100-102 \mathrm{~cm}$. 8. Heliodiscus asteriscus Haeckel, Sample 577-1-1, 5-6 cm. 9. Larcospira quadrangula Haeckel, Sample 577-1-1, 5-6 $\mathrm{cm}$. (Scale bar equals $100 \mu \mathrm{m}$; magnifications are $\times 200$.) 

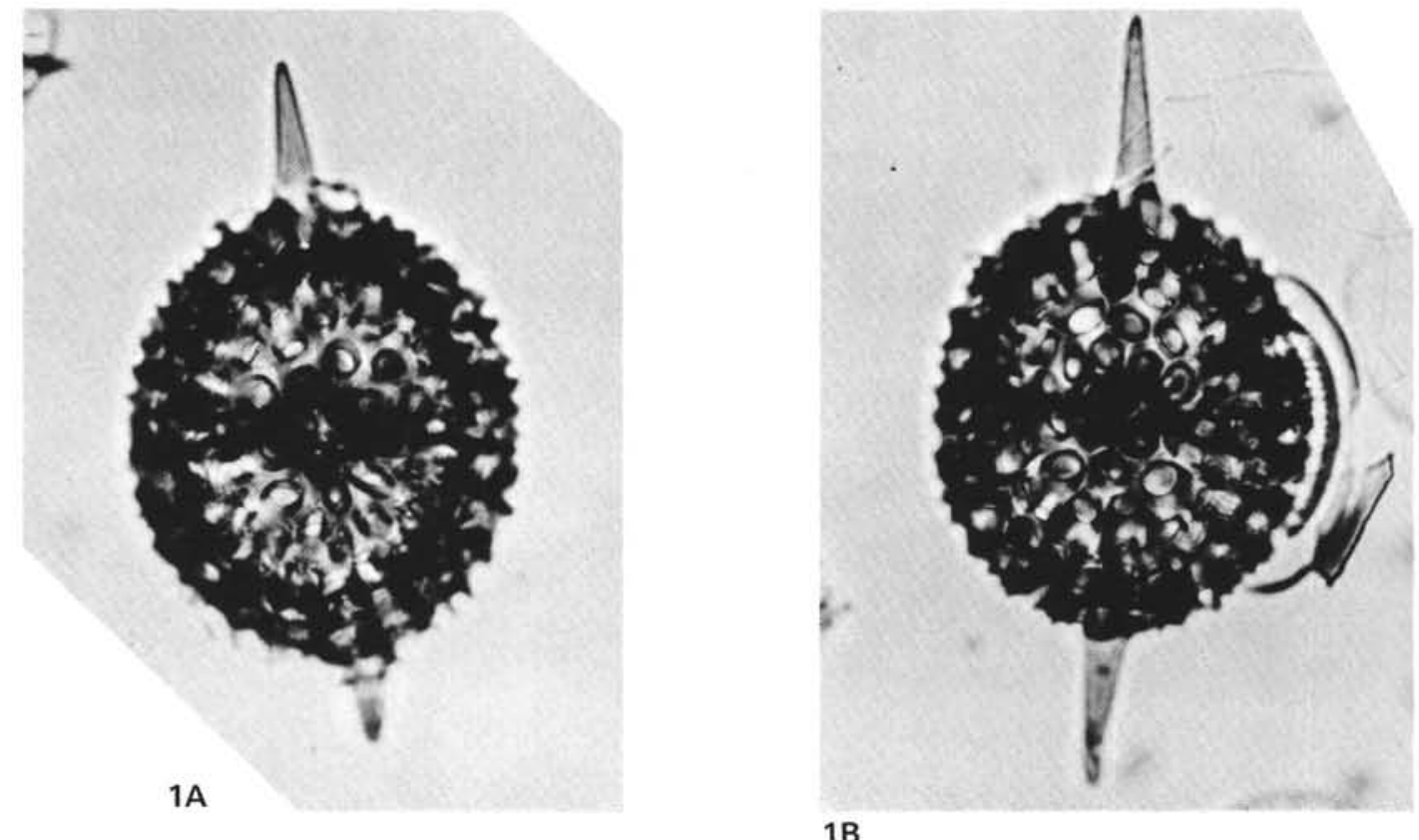

1B

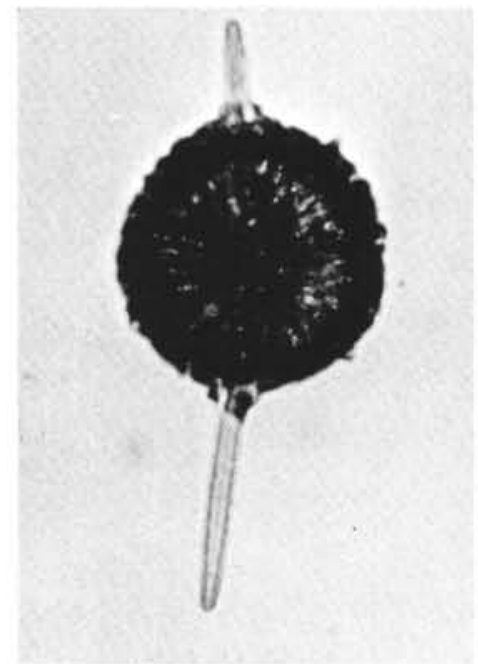

2A

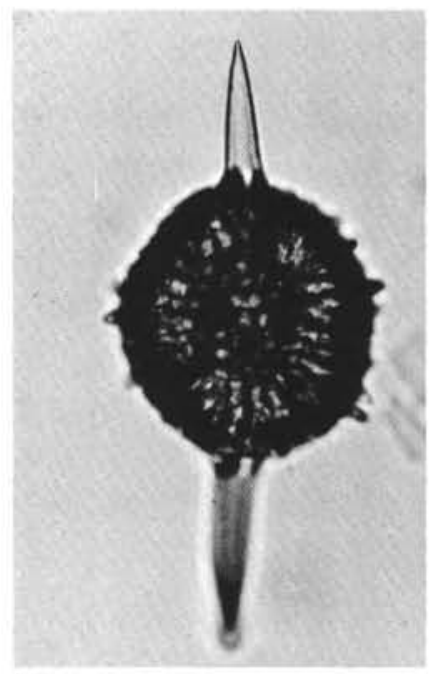

2B

Plate 4. 1A. Druppatractus acquilonius Hays, Sample 580-3-6, 105-107 cm. 1B. Druppatractus acquilonius Hays, Sample 580-3-6, 105-107 cm. 2A. Stylatractus universus Hays, Sample $578-7-1,100-102 \mathrm{~cm}$. 2B. Stylatractus universus Hays, Sample 579A-4,CC (10-12 cm). (Scale bar equals $100 \mu \mathrm{m}$; magnifications are $\times 200$.) 


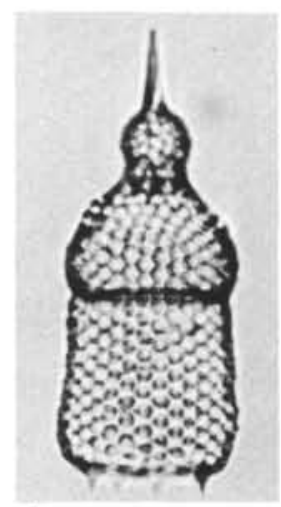

1

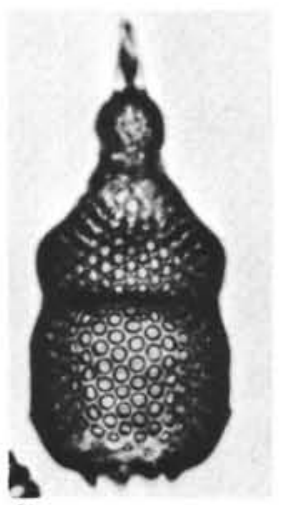

2

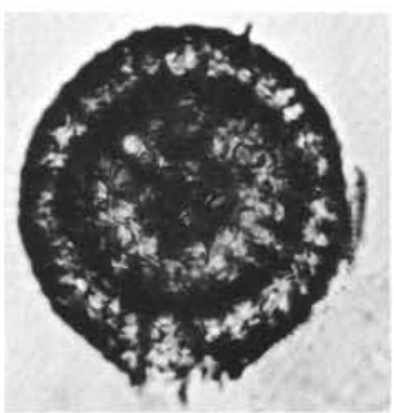

$4 \mathrm{~A}$

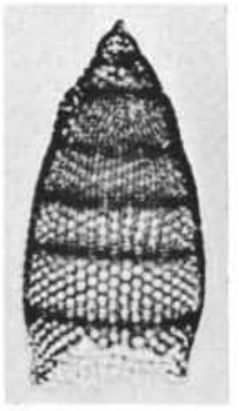

5

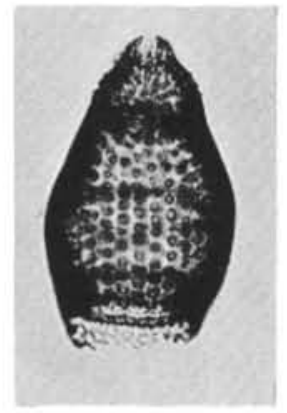

6

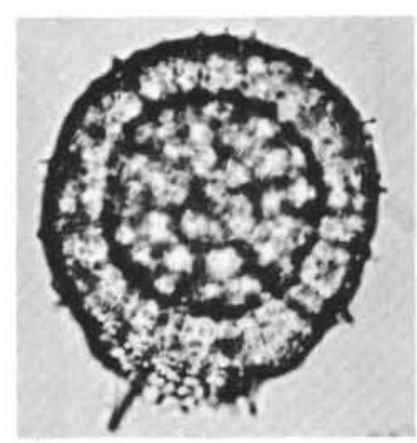

$3 A$

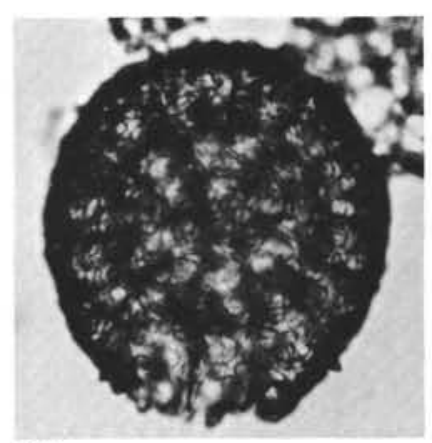

3B

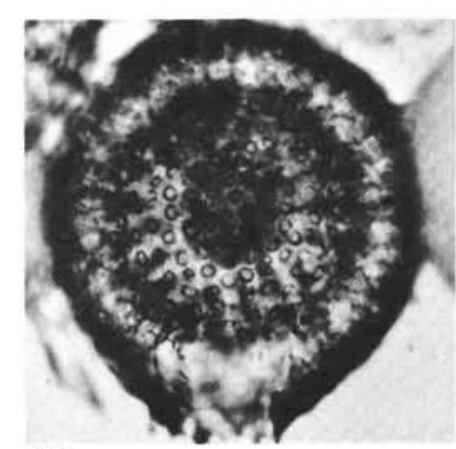

4B

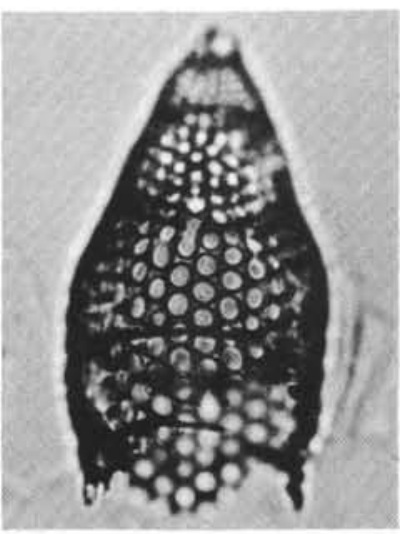

$7 \mathrm{~A}$

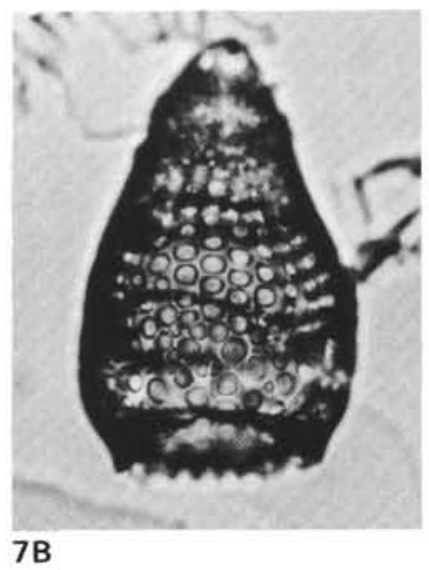

7B

Plate 5. 1. Theocorythium trachelium dianae (Haeckel), Sample $577-1-1,5-6 \mathrm{~cm}$. 2. Theocorythium trachelium trachelium (Ehrenberg), Sample 578-7-1, 100-102 cm. 3A. Sphaeropyle langii Dreyer, Sample 579A-1,CC (17-19 cm). 3B. Sphaeropyle langii Dreyer, Sample 579-2,CC (11-13 $\mathrm{cm})$. 4A. Sphaeropyle robusta Kling, Sample 579A-7-6, 70-72 cm. 4B. Sphaeropyle robusta Kling, Sample 579A-7-6, 70-72 cm. 5. Eucyrtidium acuminatum (Ehrenberg), Sample 578-1-1, 10-11 cm. 6. Eucyrtidium calvertense Martin, Sample 578-6,CC. 7A. Eucyrtidium matuyamai Hays, Sample 579A-4,CC (10-12 cm). 7B. Eucyrtidium matuyamai Hays, Sample 579A-4,CC (10-12 cm). (Scale bar equals $100 \mu$ m; magnifications are $\times 200$.) 


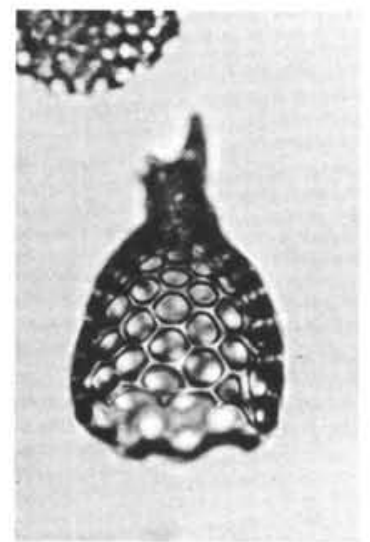

1

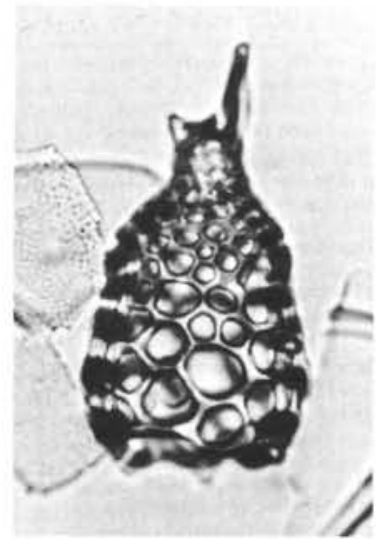

2

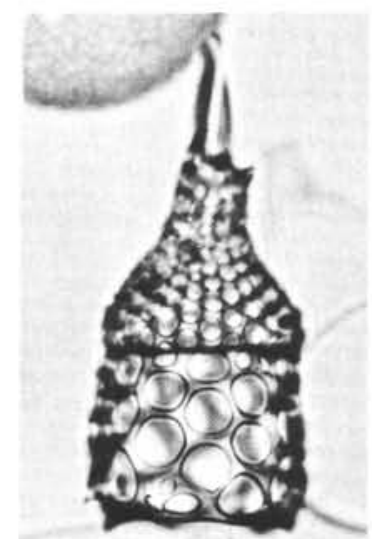

$3 A$

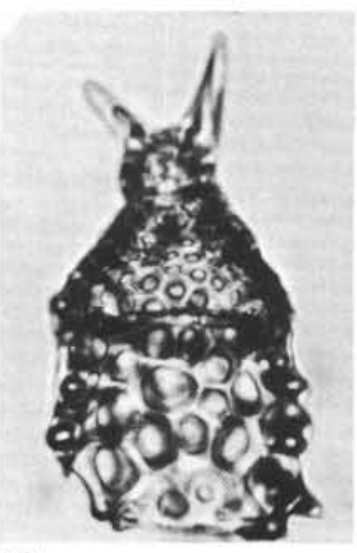

$3 B$

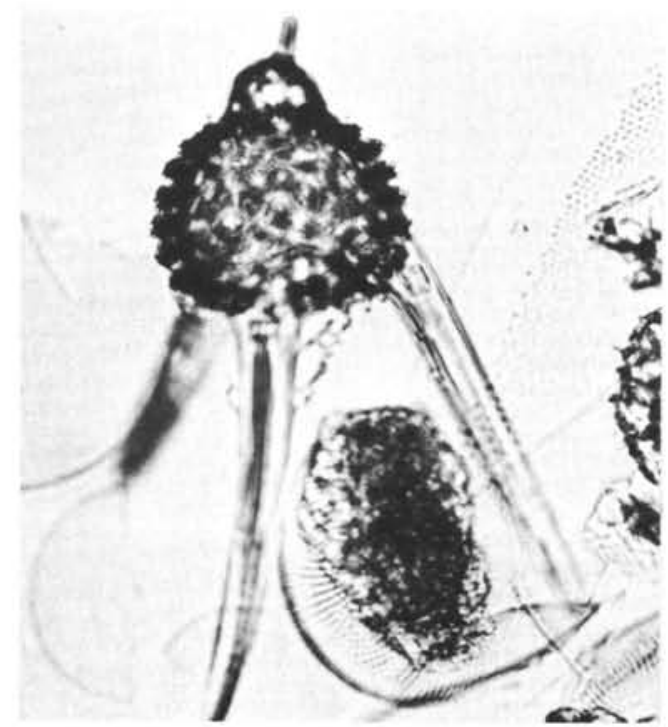

$4 \mathrm{~A}$

Plate 6. 1. Lamprocyrtis nigriniae (Caulet), Sample 579A-1,CC (17-19 cm). 2. Lamprocyrtis neoheteroporos Kling, Sample 579A-4,CC (10-12 $\mathrm{cm}$ ). 3A. Lamprocyrtis heteroporos (Hays), Sample 579A-7-6, 70-72 cm. 3B. Lamprocyrtis heteroporos (Hays), Sample 580-11,CC (20-22 $\mathrm{cm}$ ). 4A. Lychnocanoma grande (Campbell and Clark), Sample 580-3-6, 105-107 cm. 4B. Lychnocanoma grande (Campbell and Clark), Sample 580-3-6, 105-107 cm. (Scale bar equals $100 \mu \mathrm{m}$; magnifications are $\times 200$.) 


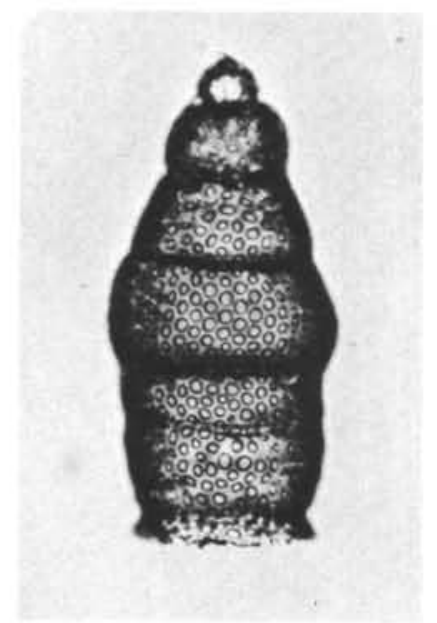

$1 \mathrm{~A}$

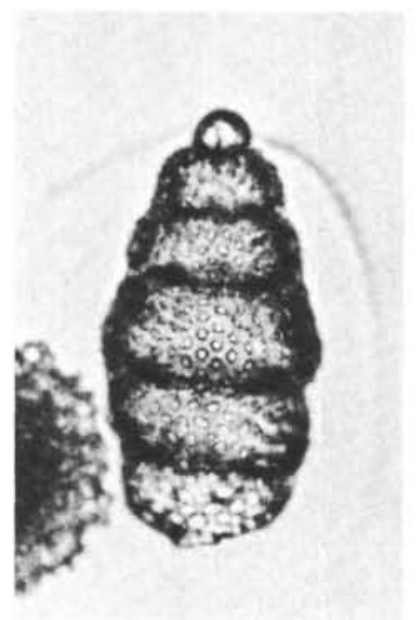

1B

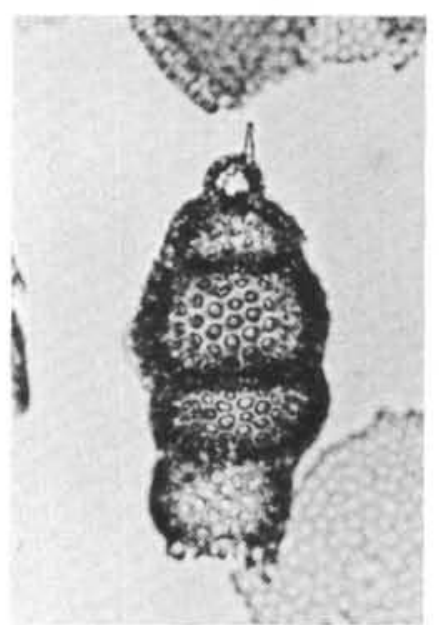

2

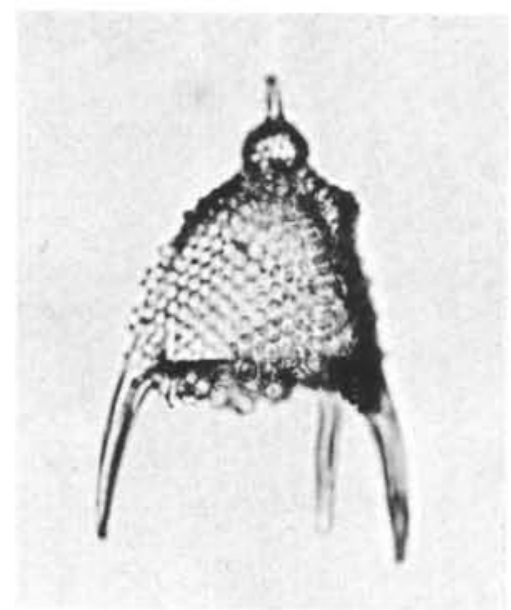

3A

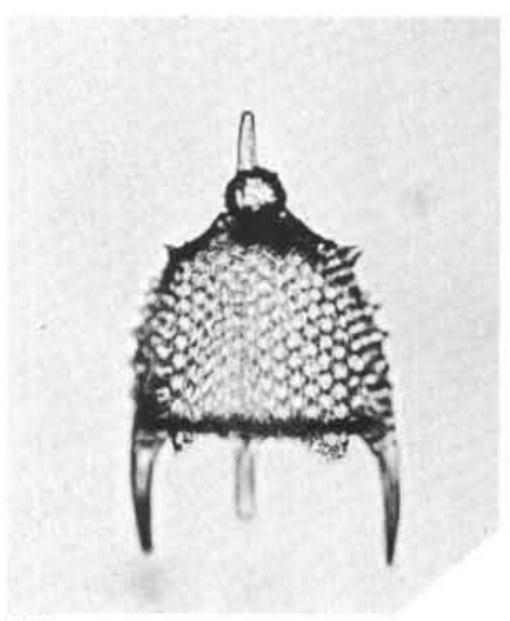

3B

Plate 7. 1A. Stichocorys peregrina (Riedel), Sample 578-11-1, 15-17 cm. 1B. Stichocorys peregrina (Riedel), Sample 581-3-1, 70-72 cm. 2. Stichocorys delmontensis (Campbell and Clark), Sample 581-7-1, 60-62 cm. 3A. Pterocanium prismatium Riedel, Sample 578-7-1, 100-102 $\mathrm{cm}$. 3B. Pterocanium prismatium Riedel, Sample 578-7-1, 100-102 cm. (Scale bar equals $100 \mu \mathrm{m}$; magnifications are $\times 200$.) 


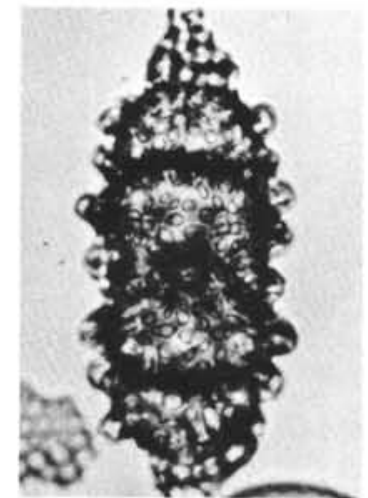

1

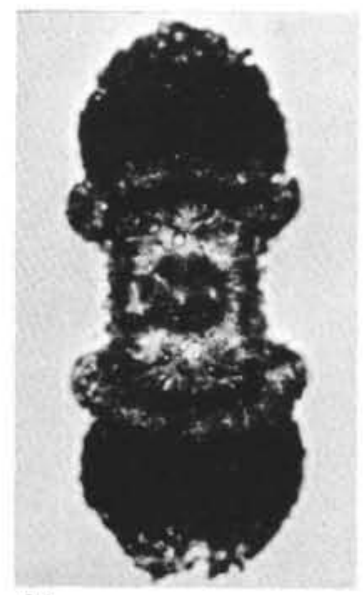

$4 \mathrm{~A}$

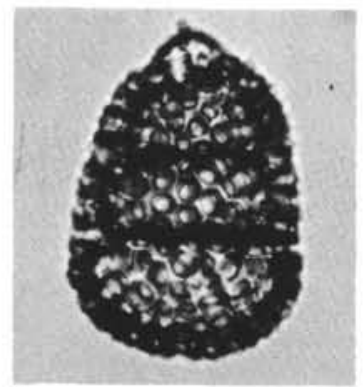

6
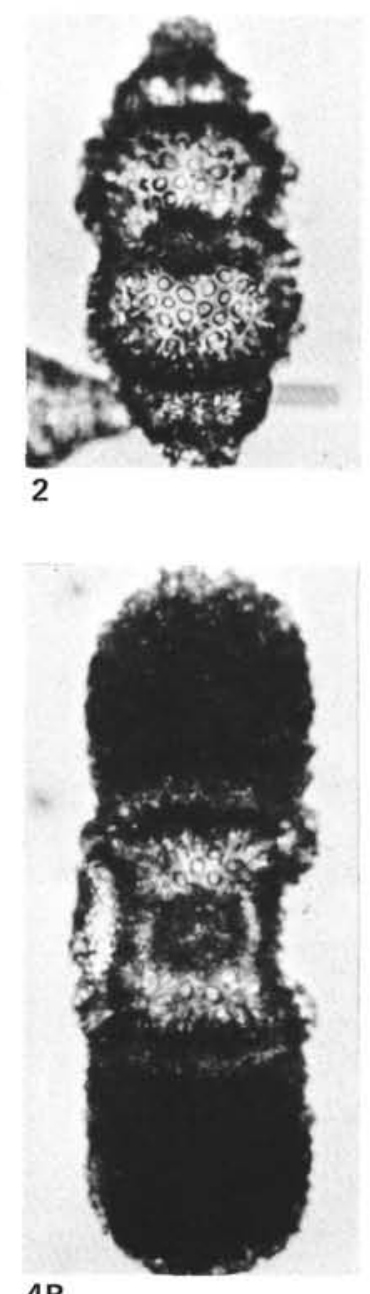

4B

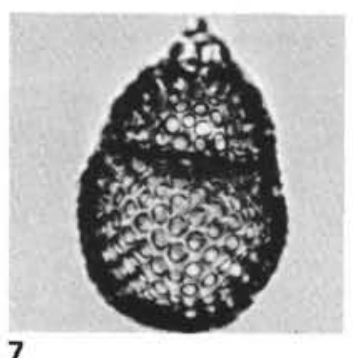

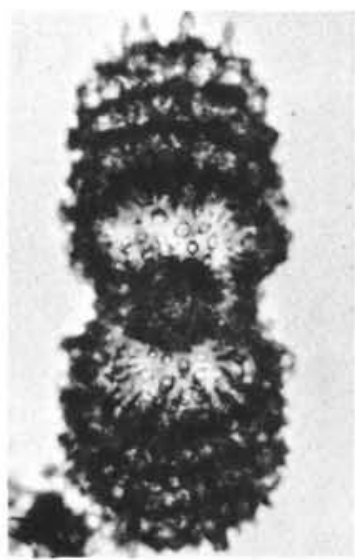

$3 \mathrm{~A}$

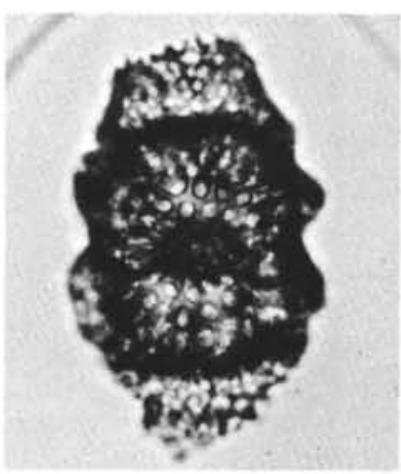

5

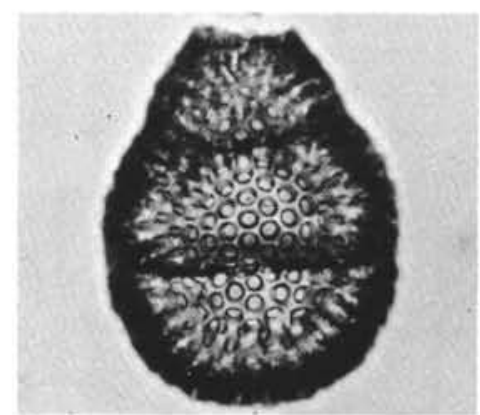

8

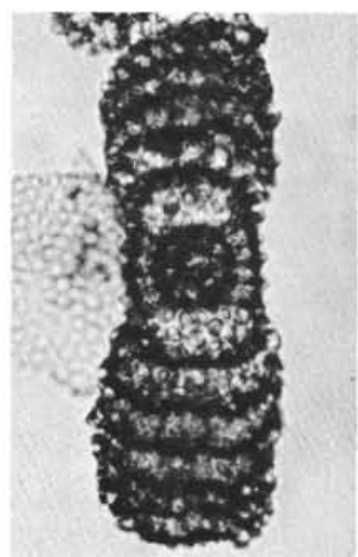

3B

Plate 8. 1. Didymocyrtis penultima (Riedel), Sample 581-6-1, 70-72 cm. 2. Didymocyrtis antepenultima (Riedel and Sanfilippo), Sample 581-71, 60-62 cm. 3A. Diartus hughesi (Campbell and Clark), Sample 581-8-1, 70-72 cm. 3B. Diartus hughesi (Campbell and Clark), Sample 5817-1, 60-62 cm. 4A. Diartus petterssoni (Riedel and Sanfilippo), Sample 581-8-1, 70-72 cm. 4B. Diartus petterssoni (Riedel and Sanfilippo), Sample 581-8-1, 70-72 cm. 5. Didymocyrtis laticonus (Riedel), Sample 581-8-1, 70-72 cm. 6. Cyrtocapsella tetrapera Haeckel, Sample 581-91, 40-42 cm. 7. Cyrtocapsella japonica (Nakaseko), Sample 581-8-1, 70-72 cm. 8. Cyrtocapsella cornuta Haeckel, Sample 581-9-1, 40-42 cm. (Scale bar equals $100 \mu \mathrm{m}$; magnifications are $\times 200$.) 